\title{
Geometric Construction of Quantum Hall Clustering Hamiltonians
}

\author{
Ching Hua Lee, ${ }^{1,2}$ Zlatko Papić, ${ }^{3,4,5}$ and Ronny Thomale ${ }^{6, *}$ \\ ${ }^{1}$ Department of Physics, Stanford University, Stanford, California 94305, USA \\ ${ }^{2}$ Institute of High Performance Computing, 1 Fusionopolis Way, Singapore 138632, Singapore \\ ${ }^{3}$ School of Physics and Astronomy, University of Leeds, Leeds LS2 9JT, United Kingdom \\ ${ }^{4}$ Perimeter Institute for Theoretical Physics, Waterloo, Ontario, Canada N2L $2 Y 5$ \\ ${ }^{5}$ Institute for Quantum Computing, Waterloo, Ontario, Canada N2L $3 G 1$ \\ ${ }^{6}$ Institute for Theoretical Physics, University of Würzburg, Am Hubland, D-97074 Würzburg, Germany \\ (Received 19 February 2015; revised manuscript received 9 August 2015; published 8 October 2015) \\ Many fractional quantum Hall wave functions are known to be unique highest-density zero modes of \\ certain "pseudopotential" Hamiltonians. While a systematic method to construct such parent Hamiltonians \\ has been available for the infinite plane and sphere geometries, the generalization to manifolds where \\ relative angular momentum is not an exact quantum number, i.e., the cylinder or torus, remains an open \\ problem. This is particularly true for non-Abelian states, such as the Read-Rezayi series (in particular, \\ the Moore-Read and Read-Rezayi $\mathbb{Z}_{3}$ states) and more exotic nonunitary (Haldane-Rezayi and Gaffnian) \\ or irrational (Haffnian) states, whose parent Hamiltonians involve complicated many-body interactions. \\ Here, we develop a universal geometric approach for constructing pseudopotential Hamiltonians that is \\ applicable to all geometries. Our method straightforwardly generalizes to the multicomponent $\mathrm{SU}(n)$ cases \\ with a combination of spin or pseudospin (layer, subband, or valley) degrees of freedom. We demonstrate \\ the utility of our approach through several examples, some of which involve non-Abelian multicomponent \\ states whose parent Hamiltonians were previously unknown, and we verify the results by numerically \\ computing their entanglement properties.
}

DOI: 10.1103/PhysRevX.5.041003

\section{INTRODUCTION}

Among the most striking emergent phenomena in condensed matter are the incompressible quantum fluids in the regime of the fractional quantum Hall $(\mathrm{QH})$ effect [1]. A key theoretical insight to understanding the many-body nature of such phases of matter was provided by Laughlin's wave function [2]. Shortly thereafter, Haldane [3] realized that the $\nu=1 / 3$ Laughlin state also occurs as an exact zero-energy ground state of a certain positive semidefinite short-range parent Hamiltonian which annihilates any electron pair in a relative angular momentum $l>1$ state, but which assigns an energy penalty for each state with $l=1$.

Haldane's construction of the parent Hamiltonian for $\nu=$ $1 / 3$ is just one aspect of a more general "pseudopotential" formalism that applies to quantum Hall systems in the infinite plane or on the surface of a sphere [3]. In those cases, the system is invariant under rotations around at least a single axis, and by virtue of the Wigner-Eckart theorem, any longrange interaction [such as Coulomb interactions projected to the Landau level (LL)] decomposes into a discrete sum of

*rthomale@physik.uni-wuerzburg.de

Published by the American Physical Society under the terms of the Creative Commons Attribution 3.0 License. Further distribution of this work must maintain attribution to the author(s) and the published article's title, journal citation, and DOI.
Subject Areas: Condensed Matter Physics

components $V_{l}$. The different components are quantized according to the relative angular momentum $l$, which is odd for spin-polarized fermions and even for spin-polarized bosons. The unique zero mode of the $V_{1}$ pseudopotential at $\nu=1 / 3$ is precisely the Laughlin state. Furthermore, it is the densest such mode because all the other states, at $\nu=1 / 3$ or any filling $\nu^{\prime}>\nu$, are separated by a finite excitation gap as indicated by overwhelming numerical evidence. In numerical simulations, it is furthermore possible to selectively turn on the magnitude of longer-range pseudopotentials $\left(V_{3}, V_{5}\right.$, etc. $)$ and verify that the Laughlin state adiabatically evolves to the exact ground state of the Coulomb interaction [4]. The corrections induced to the Laughlin state in this way are notably small (below $1 \%$ in finite systems containing about 10 particles), and the gap is maintained during the process [5]. These findings constitute an important support of Laughlin's theory.

Remarkably, the existence of pseudopotential Hamiltonians is not limited to the Laughlin states. Generically, more complicated parent Hamiltonians arise in the case of quantum trial states with non-Abelian quasiparticles, which constitute an important route towards topological quantum computation [6,7]. For example, the celebrated Moore-Read "Pfaffian" state [8], believed to describe the quantum Hall plateau at $\nu=5 / 2$ filling fraction [9], was shown to possess a parent Hamiltonian that is the shortest-range repulsive potential 
acting on three particles at a time [9-12]. This state is a member of a family of states - the parafermion ReadRezayi (RR) sequence-where the parent Hamiltonian of the $k$ th member is the shortest-range $k$-body pseudopotential [13]. Similar parent Hamiltonians have been formulated for non-Abelian $S=k / 2$ chiral-spin-liquid states [14]. More recent analysis has allowed us to resolve the structure of quantum Hall trial states interpreted as null spaces of pseudopotential Hamiltonians [15-18].

The knowledge of the parent Hamiltonian class is crucial for the complete characterization of a quantum Hall state. In certain cases, when the state can be represented as a correlator of a conformal field theory (CFT), the charged (quasihole) excitations can be constructed using the tools of the CFT [8]. (Note that such approaches become rather cumbersome for quasielectron excitations [19], and even more so in the torus geometry [20].) The tools of CFT relating wave functions to conformal blocks certainly become less useful when information about neutral excitations is needed. The knowledge of the parent Hamiltonian is thus indispensible, e.g., for estimating the neutral excitation gap of the system. In some cases, the neutral gap can be computed by the single-mode approximation [21,22], which is microscopically accurate for Abelian states [23] but requires nontrivial generalizations for the non-Abelian states [24]. Similarly, entanglement spectra may help to characterize the elementary excitations solely deduced from the ground-state wave function [25-27], but they only unfold their full strength as a complementary tool to the spectral analysis of the associated parent Hamiltonian. While a CFT underlies the structure of entanglement, conformal blocks, and clustering properties within most quantum Hall states of interest [15], it is desirable to have a general framework that complements it with a pseudopotential parent Hamiltonian.

An appealing "added value" of pseudopotentials as building blocks of parent Hamiltonians is the possibility of discovering new states by varying the considered pseudopotential terms and searching for new zero-mode ground states. This strategy is exemplified by the spinsinglet Haldane-Rezayi state [28], initially discovered as a zero mode of the "hollow core" interaction between spinful fermions at half filling. Another example is the three-body interaction of a slightly longer range than the one that gives rise to the Moore-Read state. It was found [29] that such an interaction has the densest zero-energy ground state at filling factor $\nu=2 / 5$, subsequently named the "Gaffnian" [30]. These examples illustrate that a systematic description of an operator space of parent Hamiltonians holds the promise of the discovery of previously unknown quantum Hall trial states.

All appreciable aspects of parent Hamiltonians discussed so far are independent of the geometry of the manifold in which the quantum Hall state is embedded. For several purposes, however, knowing the parent Hamiltonian on geometries without rotation symmetry, such as the torus or cylinder, is particularly desirable. For example, quantum Hall states only exhibit topological ground-state degeneracy on higher genus manifolds such as the torus [31]. Accessing the set of topologically degenerate ground states further allows us to extract the modular $\mathcal{S}, \mathcal{T}$ matrices that encode all topological information about the quasiparticles [32,33]. Furthermore, parent Hamiltonians on a cylinder or torus can be used to derive solvable models for quantum Hall states when one of the spatial dimensions becomes comparable to the magnetic length [34-39]. It has been shown that such models can be used to construct "matrix-product state" representations for quantum Hall states, and in some cases, they can be used to study the physics of "nonunitary" states and classify their gapless excitations [39-41].

A systematic construction of many-body parent Hamiltonians for quantum Hall states was first undertaken by Simon et al. for the infinite plane or sphere geometry $[42,43]$. This approach relies on the relative angular momentum, which, in this case, is an exact quantum number. As such, it cannot be applied to the cylinder, torus, or any quantum Hall lattice model such as fractional Chern insulators, for which the analogue of pseudopotentials has been developed recently [44-47].

Reference [44] has introduced the closed-form expressions for all two-body Haldane pseudopotentials on the torus and cylinder. In this work, inspired by Refs. [42-44] as the starting point of our analysis, we provide a complete framework for constructing general quantum Hall parent Hamiltonians involving $N$-body pseudopotentials, for fermions as well as bosons, in cylindric and toroidal geometries. This advance proves particularly important for the non-Abelian states, most of which necessitate many-body pseudopotentials in their parent Hamiltonian class. From the construction scheme laid out in this work, all topological properties of the non-Abelian states such as their modular matrices and topological ground-state degeneracy can now be conveniently studied from their associated toroidal parent Hamiltonian. Complementing previous results for the sphere and infinite plane, our formalism furthermore directly generalizes to multicomponent systems with an arbitrary number of "spin types" or "colors." Therefore, our construction of many-body clustered Hamiltonians not only applies to arbitrary geometries but also crucially simplifies previous approaches. We illustrate this by numerical examples, including non-Abelian multicomponent states whose parent Hamiltonians were previously unknown.

The article is organized as follows. In Sec. II, we start with a brief overview of Haldane's pseudopotential formalism from the viewpoint of clustering conditions and define our notation of generalized many-body multicomponent pseudopotentials. The clustering conditions, as well as the polynomial constraints following from them, form the core of our systematic geometric construction of 
many-body parent Hamiltonians described in Sec. III. The main result of that section is the appropriately chosen integral measure, which allows us to generate all pseudopotentials through a direct Gram-Schmidt orthogonalization. The extension to many-body parent Hamiltonians for spinful states is described in Sec. IV. Technical details of the construction are delegated to the Appendixes. In Sec. V, explicit examples of parent Hamiltonian studies are worked out in detail. We discuss spin-polarized states such as the Gaffnian, the $1 / q$ Pfaffian, and the Haffnian states, as well as spinful states such as the spin-singlet Gaffnian, the nonAbelian spin singlet (NASS), and the Halperin-permanent states. Apart from deriving the second-quantized parent Hamiltonians, we also provide extensive numerical checks of our construction using exact diagonalization and analysis of entanglement spectra. In Sec. VI, we conclude that our geometric construction of parent Hamiltonians promises ubiquitous use in the analysis of fractional quantum Hall states, and we outline a few immediate future directions.

\section{HALDANE PSEUDOPOTENTIALS}

Within a given Landau level, the kinetic energy term in the QH Hamiltonian is "quenched", i.e., it is effectively a constant. Hence, the remaining effective Hamiltonian only depends on the interaction between particles (e.g., the Coulomb potential) projected to the given Landau level [4]. In the infinite plane, the lowest Landau level (LLL) projection amounts to evaluating the matrix elements of the Coulomb interaction between the single-particle states of the form

$$
\phi_{m}(z)=z^{m} \exp \left(-|z|^{2} / 4 \ell_{B}^{2}\right),
$$

where $z$ is a complex parametrization of two-dimensional (2D) electron coordinates and $\ell_{B}=\sqrt{\hbar / e B}$ the magnetic length [Fig. 1(a)]. For simplicity, we consider a QH system in the background of a fixed (isotropic) metric [48], which allows us to write $z=x+i y$. The states in Eq. (1) are mutually orthonormal and span the basis of the LLL. There are $N_{\phi}$ of these states, which is also the number of magnetic flux quanta through the system. The above will be assumed throughout this paper, which is appropriate in strong magnetic fields when the particle-hole excitations to other Landau levels are suppressed by the large cyclotron energy gap $\hbar \omega_{c}$.

Restricting to a single LL, a large class of QH states can be classified by their clustering properties. (See Refs. $[49,50]$ for classification schemes based on clustering.) These are a set of rules that describe how the wave function vanishes as particles are brought together in space. To define the clustering rules, it is essential to first consider the problem of two particles restricted to the LLL [Fig. 1(b)]. As usual, the solution of the two-body problem

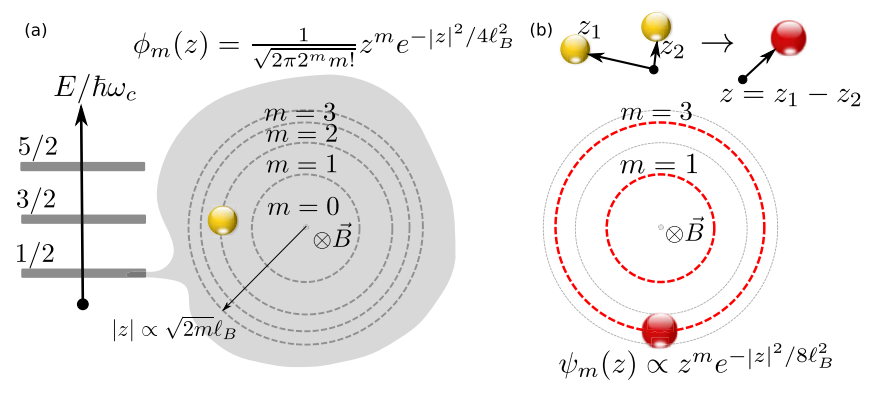

FIG. 1. (a) Landau levels and the single-particle states on the disk. The lowest LL is separated from the next lowest level by the cyclotron gap $\hbar \omega_{c}$. The wave functions of the LLL states are $\phi_{m}$, labeled by an integer $m=0,1,2 \ldots$, and Gaussian localized along the ring of radius $\sim \sqrt{m} \ell_{B}$. (b) Any two-particle state separates into a product of two wave functions, one depending on the center of mass and the other depending on the relative coordinate $z=z_{1}-z_{2}$. The relative wave function $\psi_{m}$ has an identical form to the single-particle wave function, with two important differences: Its effective magnetic length is rescaled $\left(\ell_{B} \rightarrow \ell_{B} \sqrt{2}\right)$, and the integer value of $m$ is constrained by particle statistics (assuming the spin is fully polarized); for fermions, the $m$ 's entering the relative wave function are odd (red dashed lines).

proceeds by transforming from coordinates $z_{1}, z_{2}$ into the center-of-mass (COM), $Z=\left(z_{1}+z_{2}\right) / 2$, and the relative coordinate $z \equiv z_{1}-z_{2}$ frame. In the new coordinates, the two-particle wave function decouples. As we are interested in translationally invariant problems, only the relative wave function (which depends on $z$ ) will play a fundamental role in the following analysis. For any two particles, the relative wave function turns out to have an identical form to the single-particle wave function (1),

$$
\psi_{m}\left(z \equiv z_{1}-z_{2}\right) \propto z^{m} \exp \left(-|z|^{2} / 8 \ell_{B}^{2}\right),
$$

up to the rescaling of the magnetic length $\ell_{B}$. An important difference between Eqs. (2) and (1) is the new meaning of $m$ : Since $z$ now represents the relative separation between two particles, $m$ in Eq. (2) is related to particle statistics and therefore encodes the clustering properties. For spinless electrons, $m$ in Eq. (2) is only allowed to take odd integer values since the wave function must be antisymmetric with respect to $z \rightarrow-z$, while for spinless bosons, $m$ can only be an even integer. Finally, $m$ is also the eigenvalue of the relative angular momentum for two particles $(\hbar=1)$, as we can directly confirm from

$$
L^{z}=\sum_{i} z_{i} \frac{\partial}{\partial z_{i}} .
$$

After this two-particle analysis (summarized in Fig. 1), we are in a position to introduce the notion of clustering properties for $N$-particle states. Let us pick a pair of coordinates $z_{i}$ and $z_{j}$ of indistinguishable particles in a 
many-particle wave function. We say that these particles are in a state $\psi_{m}^{i j}$, which obeys the clustering property with the power $m$ if $\psi_{m}^{i j}$ vanishes as a polynomial of total power $m$ as the coordinates of the two particles approach each other:

$$
\psi_{m}^{i j}\left(z_{i} \rightarrow z_{j}\right) \sim\left(z_{i}-z_{j}\right)^{m} .
$$

Similarly as before, we can relate the exponent $m$ to the angular momentum $L^{z}$ if the latter is a conserved quantity. Clustering conditions like this directly generalize to cases where more than two particles approach each other, with the polynomial decay also specified by a power. For example, we say that an $N$-tuple of particles $z_{1}, z_{2}, \ldots, z_{N}$ is in a state $\psi_{m}^{N}$ with total relative angular momentum $m$ if $\psi_{m}^{N}$ vanishes as a polynomial of total degree $m$ as the coordinates of $N$ particles approach each other. Fixing an arbitrarily chosen reference particle of the $N$-tuple, i.e., $z_{1}=: z_{r}$, we have

$$
\psi_{m}^{N}\left(z_{2}, \ldots, z_{N} \rightarrow z_{r}\right) \sim \prod_{j=2}^{N}\left(z_{r}-z_{j}\right)^{m_{j}},
$$

with $\sum_{j=2}^{N} m_{j}=m$ as all remaining $N-1$ particles approach the reference particle $z_{r}$. If the system is rotationally invariant about at least a single axis (such as for a disk or a sphere), it directly follows that the state in Eq. (5) is also an eigenstate of the corresponding $L_{z}$ relative angular-momentum operator with eigenvalue $m$.

The simplest illustration of the clustering condition is the fully filled Landau level. The wave function for such a state is the single Slater determinant of states $\phi_{m}\left(z_{i}\right)$ in Eq. (1). Because of the Vandermonde identity, this wave function can be expressed as

$$
\psi_{\nu=1}=\prod_{i<j}\left(z_{i}-z_{j}\right) \exp \left(-\sum_{k}\left|z_{k}\right|^{2} / 4 \ell_{B}^{2}\right) .
$$

We see that when any pair of particles $z_{i}$ and $z_{j}$ is isolated, the relevant part of the wave function is $\left(z_{i}-z_{j}\right)$. Therefore, the wave function of the filled Landau level vanishes with the exponent $m=1$ as particles are brought together. This is the minimal clustering constraint that any spinless fermionic wave function must satisfy. (As we will see in the next few sections, interesting many-body physics results from stronger clustering conditions on the wave function.)

When properly orthogonalized, the states $\left\{\psi_{m}^{N}\right\}$ form an orthonormal basis in the space of magnetic translationinvariant $\mathrm{QH}$ states. They allow us to define the $N$-body Haldane pseudopotentials [3] (PPs)

$$
U_{m}^{N}=\left|\psi_{m}^{N}\right\rangle\left\langle\psi_{m}^{N}\right|
$$

which obey the null space condition $U_{m^{\prime}}^{N} \psi_{m}^{N}=0$ for $m^{\prime} \neq m$. Since they are positive-definite, the PPs $U_{m}^{N}$ give energy penalties to $N$-body states with total relative angular momentum $m$. With a given many-body wave function, the Hamiltonian representation of $U_{m}^{N}$ will involve the sum over all $N$-tuple subsets of particles. Concrete examples of this will be given in Secs. III and V.

For a given filling fraction, a certain $\mathrm{QH}$ state can be the unique and densest ground state lying in the null space of a certain linear combination of PPs; i.e., it is annihilated by a certain number of PPs. (The requirement of being the densest state is necessary to render the finding nontrivial because it is simple, in principle, to construct additional zero modes of a given PP Hamiltonian by increasing the magnetic flux, i.e., by nucleating quasihole excitations.) The most elementary examples are the Laughlin states at $1 / m$ filling, which lie in the null space of $U_{m^{\prime}}^{2}$ for all $m^{\prime}<m$. As it also represents the densest configuration that is annihilated by the PP, the Laughlin state emerges as the unique ground state of a Hamiltonian at filling $1 / m$, $H=\sum_{m^{\prime}<m} c_{m^{\prime}} U_{m^{\prime}}^{2}$, where the coefficients are arbitrary as long as $c_{m^{\prime}}>0$. In other words, the fermionic 1/3 Laughlin state is the unique ground state of $U_{1}^{2}$, while the fermionic $1 / 5$ state is the unique ground state of any linear combination of $U_{1}^{2}$ and $U_{3}^{2}$ with positive weights. Note that the PPs of even $m^{\prime}$ are precluded by fermionic antisymmetry. For short-ranged two-body interactions on the disk where the degree of the clustering polynomial $m$ coincides with the exact relative angular momentum $l$, the traditional notation by Haldane [3] relates to ours via $V_{l} \equiv U_{m}^{2}$. As will be explicitly discussed in Sec. V, many more exotic states can be realized as the highest-density ground states of combinations of PPs involving $N \geq 2$ bodies.

In view of other geometries than disk or sphere types, one question immediately arises: Is there any hope of defining PPs in the absence of continuous rotation symmetry and hence with no exact relative angular-momentum quantum number? This question is natural because one of the popular choices for the gauge of the magnetic fieldthe Landau gauge-is only compatible with periodic boundary conditions along one or both directions in the plane, which breaks continuous rotational symmetry.

The answer to the above question is affirmative, which is the central message of this paper. This result is mainly because the clustering conditions, i.e., the polynomial exponent $m$, are more fundamental than their interpretation as the relative angular momentum. The clustering conditions are short-distance properties: Their support is the area associated with the fundamental droplet of $N$ particles $\sim N \times 2 \pi \ell_{B}^{2}$. Assuming a one-to-one correspondence between a clustering power and a pseudopotential, the PPs should be independent of the specific geometry as long as they act in a manifold that is homogeneous and much larger than the fundamental $N$-particle droplet. This viewpoint has been confirmed by the explicit constructions of quantum Hall trial wave functions. For instance, the successful generalization of the Laughlin wave function to 
the torus, given in the classic paper by Haldane and Rezayi [51], has demonstrated that its short-distance properties are identical to its original version defined on the disk. The states in both geometries are uniquely characterized by their clustering properties and are locally indistinguishable; their main difference lies in the global properties, e.g., the fact that the torus Laughlin state is $m$-fold degenerate because of its invariance under the COM translation. This degeneracy is of intrinsically topological origin [31]. At filling $\nu=p / q$, where $p, q$ are relative coprime integers, the quantum Hall state on a torus is invariant under the translation that moves every particle by $q$ orbitals [see Fig. 3(c) below]. This symmetry guarantees an exact $m$-fold degeneracy of the Laughlin $\nu=1 / m$ state on the torus [52]. Additional topological degeneracies can arise for more complicated (non-Abelian) states $[8,10]$.

In the following, we assume there exists, in general, a well-defined deformation of the null space specified by a planar QH trial wave function to the multidimensional null space specified by the associated set of topologically degenerate $\mathrm{QH}$ ground states on the torus. What we are then interested in is finding a suitable deformation of the planar Laplacian, whose bilinear form is the known planar parent Hamiltonian composed of the spherical PPs, to the toroidal Laplacian, whose bilinear form is the toroidal Hamiltonian. In the following sections, we solve this problem by what we refer to as the "geometric construction" of pseudopotentials.

\section{GEOMETRIC CONSTRUCTION OF PSEUDOPOTENTIALS: SPINLESS CASE}

We describe the construction of generic QH pseudopotential Hamiltonians with a perpendicular magnetic field applied to the $2 \mathrm{D}$ electron gas. We assume the field to be sufficiently strong such that the spin is fully polarized and that there are no further internal degrees of freedom for the particles. We first introduce a suitable single-particle basis for generic $N$-body interactions that obeys the magnetic translation symmetry and conserves the COM momentum. Next, we show how the explicit functional form of Haldane PPs in Sec. II can be easily obtained from geometric principles and symmetry. We constrain ourselves to singlecomponent PPs in this section and generalize our construction to multicomponent (spinful) PPs in Sec. IV.

\section{A. Basis choice}

A well-developed pseudopotential formalism is available in the literature $[3,30]$ for the infinite plane or the sphere, where the $z$ component of angular momentum is conserved. A different approach to pseudopotential Hamiltonian construction, however, is needed when the system is no longer invariant under continuous rotations around the $z$ axis. This can occur when periodic boundary conditions are imposed (Fig. 2), either along one direction (cylinder geometry) or

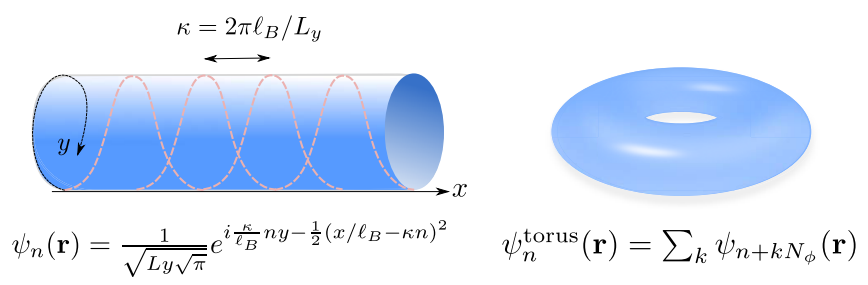

FIG. 2. Boundary conditions considered in this paper, compatible with Landau gauge: cylinder (left diagram) and torus (right diagram). The associated single-particle states $\psi_{n}(\mathbf{r})$ are arranged along a one-directional (1D) chain, with the parameter $\kappa=2 \pi \ell_{B} / L_{y}$ controlling the distance between nearest-neighbor orbitals.

both directions (torus). Under periodic boundary conditions in one direction (say, $\hat{y}$ ), the single-particle Hilbert space is spanned by the Landau gauge basis wave function labeled by $n$ :

$$
\psi_{n}(x, y) \equiv\left\langle\vec{r}\left|c_{n}^{\dagger}\right| 0\right\rangle \propto e^{i\left(\kappa / \ell_{B}\right) n y} e^{\left.-\frac{1}{2}\left(x / \ell_{B}\right)-\kappa n\right]^{2}}
$$

where $\ell_{B}=\sqrt{\hbar / e B}$ is the magnetic length and $c_{n}^{\dagger}$ is the second-quantized operator that creates a particle in the state $|n\rangle$. The parameter $\kappa=2 \pi \ell_{B} / L_{y}$ sets the effective separation between the one-body states in the $x$ direction, as each one-body state is a Gaussian packet approximately localized around $\kappa n$ in the $x$ direction (Fig. 2).

Because of this simple one-parameter labeling of the one-body states, an $\mathrm{N}$-body interaction matrix element is labeled by $2 N$ indices. Additional constraints on its functional form arise when we consider interactions projected to a given Landau level (Fig. 3). Because of the magnetic translation invariance, the interactions projected to a Landau level only allow for scattering that conserves total momentum or, equivalently, leaves the COM of the particles fixed. For example, the process in Fig. 3(a) is allowed, but the one in Fig. 3(b) is forbidden. This special structure in the interaction Hamiltonian gives rise to the symmetry under many-body translations that shifts every particle by $q$ orbitals at filling $\nu=p / q$ [Fig. 3(c)]. This is

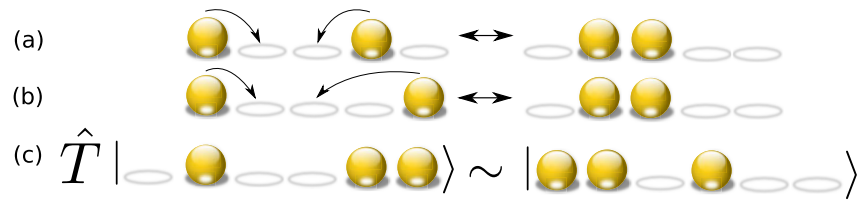

FIG. 3. Scattering schemes and symmetries in a Landau level. Preservation of COM allows a cluster of particles to scatter according to line (a), but forbids the scattering according to line (b). (c) The many-particle translation operator $\hat{T}$ acts on a given configuration by moving every particle $q$ orbitals to the right (in this example, $q=2$ ). Many-body states at filling $\nu=p / q$ are invariant under this symmetry, which gives rise to the topological degeneracy equal to $q$. 
the symmetry that underlies the topological ground-state degeneracy.

The matrix elements of any interaction $V\left(\mathbf{r}_{1}, \ldots, \mathbf{r}_{N}\right)$ projected to a Landau level are given by

$$
\begin{aligned}
V_{\left\{n_{j}\right\},\left\{n_{j}^{\prime}\right\}}= & \int d \mathbf{r}_{1}, \ldots, d \mathbf{r}_{N} \\
& \times\left(\prod_{j} \psi_{n_{j}^{\prime}}^{*}\left(\mathbf{r}_{j}\right) \psi_{n_{j}}\left(\mathbf{r}_{j}\right)\right) V\left(\mathbf{r}_{1}, \ldots, \mathbf{r}_{N}\right),
\end{aligned}
$$

which corresponds to the second-quantized Hamiltonian

$$
\begin{aligned}
& H=\sum_{\alpha} \sum_{n} b_{n}^{\alpha \dagger} b_{n}^{\alpha}, \\
& b_{n}^{\alpha} \propto \sum_{\sum_{j} n_{j}=n} p_{\alpha}\left(\kappa \bar{n}_{1}, \ldots, \kappa \bar{n}_{N}\right) e^{-\left(\kappa^{2} / 2\right) \sum_{j=1}^{N} \bar{n}_{j}^{2}} c_{n_{1}}, \ldots, c_{n_{N}} .
\end{aligned}
$$

Here, $\bar{n}_{j}=n_{j}-n / N$ denotes the orbital of particle $j$ with respect to the COM, $n=n_{1}+\cdots+n_{N}$, and $p_{\alpha}$ is a polynomial in $N$ variables $\kappa \bar{n}_{j}$. The index $\alpha$, as will be clear from the explicit construction of $p_{\alpha}$ below, specifies the degree of the polynomial and, for a multicomponent state discussed in Sec. IV, its spin sector. The polynomial $p_{\alpha}$ can be chosen to be real, as will be evident from its geometric construction later. From now on, $n$ will refer to the COM and not the index of a single particle previously appearing in Eq. (8). $c_{n_{j}}^{\dagger}$ is the same operator as in Eq. (8), creating the $j$ th particle in state $\left|n_{j}\right\rangle$. The Hamiltonian thus consists of a product sum over all positions of the COM $n$, as well as all polynomial degrees $\alpha$.

Equations (9) and (10) represent a general translationally invariant Hamiltonian projected to a Landau level. This Hamiltonian has a rather special form: It decomposes into a linear combination of positive-definite operators $b_{n}^{\alpha \dagger} b_{n}^{\alpha}$, such that the form factor $p_{\alpha} e^{-\left(\kappa^{2} / 2\right) \sum_{j=1}^{N} \bar{n}_{j}^{2}}$ in each $b_{n}^{\alpha}$ depends only on the relative coordinates of particles. Any short-range Hamiltonian can be explicitly expressed in this form $[34,35,44]$, which reveals its Laplacian structure.

Physically, Landau-level-projected Hamiltonians can be visualized as a long-range interacting 1D chain (Fig. 3). The interaction terms can be interpreted as long-range (though Gaussian-suppressed) hopping processes labeled by $\alpha$. For each $\alpha, N$ particles "hop" from sites $n_{j}$ to sites $n_{j}^{\prime}$, $j=1, \ldots, N$ according to a COM-independent amplitude given by $p_{\alpha}\left(\kappa \bar{n}_{1}, \ldots, \kappa \bar{n}_{N}\right) e^{-\left(\kappa^{2} / 2\right) \sum_{j=1}^{N} \bar{n}_{j}^{2}}$, such that the initial and final COM $n$ remains unchanged [Fig. 3(c)]. Note that although we use the term "hopping," there is no clear distinction between hopping and "interaction" in our case, as opposed to the Hubbard model. Rather, hopping is designated for any interaction term that is purely quantum (i.e., not of Hartree form). The goal of the remainder of this paper is to show how to systematically construct the polynomial amplitudes $p_{\alpha}$ that need to be inserted into Eq. (10) to obtain the parent Hamiltonian for the desired quantum Hall state.

\section{B. Geometric derivation}

We now specialize Eq. (9) to $N$-body interactions that are Haldane PPs $U_{m}^{N}$. One appealing feature of the secondquantized form of Eq. (9) is that we can construct the desired PPs from symmetry principles alone, without referring to the first-quantized form of the interaction $V\left(\mathbf{r}_{1}, \ldots, \mathbf{r}_{N}\right)$.

The many-body PPs are, by definition, supposed to project onto orthogonal subspaces labeled by $m$, where, if applicable, $m$ denotes the relative angular momentum:

$$
U_{m^{\prime}}^{N} U_{m}^{N}=\left|\psi_{m^{\prime}}^{N}\right\rangle\left\langle\psi_{m^{\prime}}^{N}|| \psi_{m}^{N}\right\rangle\left\langle\psi_{m}^{N}\right|=U_{m}^{N} \delta_{m^{\prime} m} .
$$

This requires that

$$
\begin{aligned}
\left\langle 0\left|b_{n}^{m^{\prime} \dagger} b_{n}^{m}\right| 0\right\rangle \propto & \sum_{\bar{n}_{1}+\cdots+\bar{n}_{N}=0} p_{m^{\prime}}\left(\kappa \bar{n}_{1}, \ldots, \kappa \bar{n}_{N}\right) \\
& \times p_{m}\left(\kappa \bar{n}_{1}, \ldots, \kappa \bar{n}_{N}\right) \exp \left(-\kappa^{2} \sum_{j=1}^{N} \bar{n}_{j}^{2}\right) \\
= & \delta_{m^{\prime} m} .
\end{aligned}
$$

If $\psi_{m}^{N}$ vanishes with $m$ th total power as $N$ particles approach each other, the polynomial $p_{m}$ must be of degree $m$. Hence, the PPs will be completely determined once we find a set of polynomials $\left\{p_{m}\right\}$ such that (1) $p_{m}$ is of total degree $m$, (2) $p_{m}$ has the correct symmetry property under exchange of particles, i.e., is totally (anti)symmetric for bosonic (fermionic) particles, and (3) the $p_{m}$ 's are orthonormal under the inner product measure

$$
\begin{aligned}
\left\langle p_{m^{\prime}}, p_{m}\right\rangle= & \sum_{\bar{n}_{1}+\cdots+\bar{n}_{N}=0} p_{m^{\prime}}\left(\kappa \bar{n}_{1}, \ldots, \kappa \bar{n}_{N}\right) \\
& \times p_{m}\left(\kappa \bar{n}_{1}, \ldots, \kappa \bar{n}_{N}\right) \exp \left(-\kappa^{2} \sum_{j=1}^{N} \bar{n}_{j}^{2}\right) .
\end{aligned}
$$

Using the barycentric coordinates (Appendix A) to represent the tuple $\left(\bar{n}_{1}, \ldots, \bar{n}_{N}\right)$,

$$
\begin{aligned}
\left\langle p_{m^{\prime}}, p_{m}\right\rangle \approx & \int_{\mathbb{R}^{N-1}} d W d \Omega p_{m^{\prime}}(W, \Omega) p_{m}(W, \Omega) \\
& \times \exp \left(-\frac{N-1}{N} W^{2}\right) J_{N-1}(W, \Omega) \\
= & \delta_{m^{\prime} m},
\end{aligned}
$$

where $W$ and $\Omega$ are the radial and angular coordinates of the vector $\vec{x} \in \mathbb{R}^{N-1}$ representing the tuple $\left(\bar{n}_{1}, \ldots, \bar{n}_{N}\right)$, and 
$J_{N-1}$ is the Jacobian for the spherical coordinates in $\mathbb{R}^{N-1}$. We have exploited the magnetic translation symmetry of the problem in quotienting out the COM coordinate $n$. (It is desirable to quotient out $n=\sum_{j}^{N} n_{j}$ since $n$ takes values on an infinite set when the particles lie on the 2D infinite plane, and that complicates the definition of the inner product measure.) Each quotient space is most elegantly represented as an $N-1$ simplex in barycentric coordinates, where particle permutation symmetry (or subgroups of it) is manifest. Explicitly, the set of $\bar{n}_{j}$ can be encoded in the vector

$$
\vec{x}=\kappa \sum_{j=1}^{N} \bar{n}_{j} \vec{\beta}_{j},
$$

where the $N$ basis vectors $\left\{\vec{\beta}_{j}\right\}$ form a set that spans $\mathbb{R}^{N-1}$. A configuration $\left(\bar{n}_{1}, \ldots, \bar{n}_{N}\right)$ is uniquely represented by a point $\vec{x} \in \mathbb{R}^{N-1}$ that is independent of $n=\sum_{j}^{N} n_{j}$. Since $\vec{x}$ should not favor any particular $\bar{n}_{j}$, any pair of vectors in the basis $\left\{\vec{\beta}_{j}\right\}$ must form the same angle with each other. Specifically,

$$
\vec{\beta}_{j} \cdot \vec{\beta}_{k}=\frac{N}{N-1} \delta_{j k}-\frac{1}{N-1},
$$

so that each vector points at the angle of $\pi-\cos ^{-1}(1) /$ $(N-1)$ from another vector.

With this parametrization, the Gaussian factor reduces to the simple form

$$
W^{2}=|\vec{x}|^{2}=\frac{N}{N-1} \kappa^{2} \sum_{j=1}^{N} \bar{n}_{j}^{2} .
$$

Further mathematical details can be found in the examples that follow, as well as in Appendix A.

The integral approximation in Eq. (14) becomes exact in the infinite plane limit and is still very accurate for values of $m$ where the characteristic interparticle separation is smaller than the smallest of the two linear dimensions of the $\mathrm{QH}$ system. In the following, we assume this to be the case; otherwise, there can be significant effects from the interaction of a particle with its periodic images. This was systematically studied in the Appendix of Ref. [44] for $N=2$. We note that the approximation in Eq. (14) does not affect the exact zero-mode property of the trial Hamiltonians constructed below.

\section{Orthogonalization}

We are now ready to evaluate the pseudopotentials. To find second-quantized PPs with relative angular momentum $m$, one needs to follow the rules listed in Table I. In the following, we shall execute this recipe explicitly for $N=2,3$, and, to some extent, $N=4$-body interactions.
TABLE I. Summary of the PP construction for spinless particles. Examples of this procedure are given in Sec. V.

(1) Write down the allowed "primitive" polynomials [30,42,43] of degree $m^{\prime} \leq m$ consistent with the symmetry of the particles.

(2) Orthogonalize this set of primitive polynomials according to the inner product measure in Eq. (14).

\section{Two-body case}

For two-body interactions, we have

$$
\left\langle p_{m^{\prime}}, p_{m}\right\rangle=\int_{-\infty}^{\infty} p_{m^{\prime}}(W) p_{m}(W) e^{-\frac{1}{2} W^{2}} d W
$$

where $W=-2 \kappa \bar{n}_{1}=2 \kappa \bar{n}_{2}$. For this $N=2$ case, we have allowed $W$ to take negative values as the angular direction spans the $1 \mathrm{D}$ circle, which consists of just two points. The primitive polynomials for bosons are $\left\{1, W^{2}, W^{4}, W^{6}, \ldots\right\}$, while those for fermions are $\left\{W, W^{3}, W^{5}, \ldots\right\}$. After performing the Gram-Schmidt orthogonalization procedure, the two-body PPs are found to be $U_{m}^{2} \propto \kappa^{3} \sum_{n} b_{n}^{m \dagger} b_{n}^{m}$, where

$b_{n}^{m}=\sum_{\bar{n}_{1}+\bar{n}_{2}=0} p_{m}\left(\kappa \bar{n}_{1}, \kappa \bar{n}_{2}\right) e^{-\frac{1}{2} \kappa^{2}\left(\bar{n}_{1}^{2}+\bar{n}_{2}^{2}\right)} c_{n / 2+\bar{n}_{1}} c_{n / 2+\bar{n}_{2}}$,

$n / 2+\bar{n}_{j}$ are integers, and $p_{m}$ is the $m$ th-degree Hermite polynomial given in Table II. In particular, we recover the Laughlin $\nu=1 / 2$ bosonic or $\nu=1 / 3$ fermionic state for $m=0$ or $m=1$, respectively.

One can easily check that PPs become more delocalized in $W$ space as $m$ increases. Indeed,

$$
\begin{aligned}
\kappa^{2}\left\langle\sum_{j} \bar{n}_{j}^{2}\right\rangle & =\frac{1}{2}\left\langle W^{2}\right\rangle=\frac{1}{2} \int_{-\infty}^{\infty} p_{m}(W) W^{2} e^{-\frac{1}{2} W^{2}} d W \\
& =m+\frac{1}{2},
\end{aligned}
$$

which is reminiscent of the interpretation of $m$ as the angular momentum of a pair of particles in rotationally invariant geometries (Fig. 1). Obtaining the pseudopotentials in this second-quantized form is highly advantageous. In particular, note that this construction is free from ambiguities in the choice of $V\left(\mathbf{r}_{1}, \ldots, \mathbf{r}_{N}\right)$ since several possible real-space interactions, e.g., those of the TrugmanKivelson type [53], can all be grouped into the same $m$ sector. This is discussed in more detail in Appendix B.

\section{Three-body case}

For $N=3$, the inner product measure takes the form

$\left\langle p_{m^{\prime}}, p_{m}\right\rangle=\int_{0}^{\infty} \int_{0}^{2 \pi} p_{m^{\prime}}(W, \theta) p_{m}(W, \theta) e^{-\frac{2}{3} W^{2}} W d \theta d W$, 
TABLE II. Representative polynomials $p_{m}$ for the first few $N=2$-body PPs for bosons and fermions. $p_{m}(W) e^{-W^{2} / 4}$ represents the probability amplitude that two particles $W / \kappa$ sites apart along a chain are involved in a two-body hopping.

\begin{tabular}{lcc}
\hline \hline$m$ & Bosonic $p_{m}(W)$ & Fermionic $p_{m}(W)$ \\
\hline 0 & 1 & 0 \\
1 & 0 & $W$ \\
2 & $(1 / \sqrt{2 !})\left(-1+W^{2}\right)$ & 0 \\
3 & 0 & $(1 / \sqrt{3 !})\left(-3+W^{2}\right) W$ \\
4 & 0 \\
5 & $(1 / \sqrt{4 !})\left(3-6 W^{2}+W^{4}\right)$ & $(1 / \sqrt{5 !})\left(15-10 W^{2}+W^{4}\right) W$ \\
6 & 0 & 0 \\
7 & $(1 / \sqrt{6 !})\left(-15+45 W^{2}-15 W^{4}+W^{6}\right)$ & $(1 / \sqrt{7 !})\left(-105+105 W^{2}-21 W^{4}+W^{6}\right) W$ \\
\hline
\end{tabular}

with

$$
\begin{aligned}
& \bar{n}_{1}=\frac{2 W}{3 \kappa} \cos \theta, \\
& \bar{n}_{2}=\frac{W}{3 \kappa}(\sqrt{3} \sin \theta-\cos \theta), \\
& \bar{n}_{3}=\frac{W}{3 \kappa}(-\sqrt{3} \sin \theta-\cos \theta) .
\end{aligned}
$$

Each of the $\bar{n}_{j}$ 's are treated on equal footing, as one can easily check graphically. The above expressions are the simplest nontrivial cases of the general expressions for barycentric coordinates found in Eqs. (A3)-(A5) of Appendix A.

The bosonic primitive polynomials are made up of elementary symmetric polynomials $S_{1}, S_{2}, S_{3}$ in the variables $\kappa \bar{n}_{j}=\kappa\left(n_{j}-n / N\right)$. Since $S_{1}=\kappa \sum_{j} \bar{n}_{j}=0$, the only two symmetric primitive polynomials are

$-S_{2}=-\kappa^{2} \sum_{i<j} \bar{n}_{i} \bar{n}_{j}=\frac{S_{1}^{2}-2 S_{2}}{2}=\frac{\kappa^{2}}{2} \sum_{i} \bar{n}_{i}^{2}=\frac{1}{3} W^{2}$

and

$$
\begin{aligned}
Y=S_{3} & =\kappa^{3} \prod_{i} \bar{n}_{i} \\
& =\frac{\kappa^{3}}{27}\left(2 n_{1}-n_{2}-n_{3}\right)\left(2 n_{2}-n_{3}-n_{1}\right)\left(2 n_{3}-n_{1}-n_{2}\right) \\
& =\frac{2}{27} W^{3} \cos 3 \theta
\end{aligned}
$$

The fermionic primitive polynomials are totally antisymmetric and can always be written $[42,43]$ as a symmetric polynomial multiplied by the Vandermonde determinant

$$
\begin{aligned}
A & =\kappa^{3}\left(n_{1}-n_{2}\right)\left(n_{2}-n_{3}\right)\left(n_{3}-n_{1}\right) \\
& =\kappa^{3}\left(\bar{n}_{1}-\bar{n}_{2}\right)\left(\bar{n}_{2}-\bar{n}_{3}\right)\left(\bar{n}_{3}-\bar{n}_{1}\right) \\
& =-\frac{2}{3 \sqrt{3}} W^{3} \sin 3 \theta .
\end{aligned}
$$

Note that $W^{2}$ is of degree 2, while $A$ and $Y$ are of degree 3. All of them are independent of the COM coordinate $n$, as they should be. $N=3$ PPs were derived in Ref. [44] through explicit integration, and the approach discussed here considerably simplifies those computations by exploiting symmetry.

To generate the fermionic (bosonic) PPs up to $U_{m}^{3}$, we need to orthogonalize the basis consisting of all possible (anti)symmetric primitive polynomials up to degree $m$. For instance, the first seven (up to $m=9$ ) three-body fermionic PPs are generated from the primitive basis $\left\{A, A W^{2}, A Y, A W^{4}, A Y W^{2}, A Y^{2}, A W^{3}\right\}$. Note that the last two basis elements both contribute to the $m=9$ PP sector.

The three-body PPs are found to be $U_{m}^{3} \propto \kappa^{3} \sum_{n} b_{n}^{m \dagger} b_{n}^{m}$, where

$$
b_{n}^{m}=\sum_{n_{1}+n_{2}+n_{3}=n} p_{m}(W, Y, A) e^{-\frac{1}{3} W^{2}} c_{n_{1}} c_{n_{2}} c_{n_{3}},
$$

with the polynomials $p_{m}$ listed in Table III. These results are fully compatible with those from Ref. [42]. As mentioned, there can be more than one (anti)symmetric polynomial of the same degree for sufficiently large $m$. This leads to the degenerate PP subspace, a specific example of which is presented in Sec. VA 2.

\section{3. $N \geq$ 4-body case}

For general PPs involving $N$ bodies, the inner product measure takes the form 
TABLE III. The polynomials $p_{m}$ for $N=3$-body PPs for bosons and fermions up to $m=9$. Note that there is more than one possible PP for larger $m$ since there are multiple ways to build a homogeneous (anti)symmetric polynomial from the primitive and elementary symmetric polynomials. For instance, with $Y$ and $A$ defined in Eqs. (24) and (25), there are two possible bosonic PPs for $m=6$ since there are two ways $\left(W^{6}\right.$ and $\left.Y^{2}\right)$ to build a homogeneous 6-degree polynomial from elementary symmetric polynomials. For the fermionic cases, we have explicitly kept only one factor of $A$ since even powers of $A$ can be expressed in terms of $W^{2}$ and $Y$. For all cases, the mean-square spread of the PPs also increases linearly with $m$, i.e., $\kappa^{2}\left\langle\sum_{j}^{3} \bar{n}_{j}^{2}\right\rangle=m+1$ [compare with the two-body case in Eq. (20)].

\begin{tabular}{llc}
\hline \hline$m$ & Bosonic $p_{m}(W, Y)$ & Fermionic $p_{m}(W, Y, A)$ \\
\hline 0 & 1 & 0 \\
1 & 0 & 0 \\
2 & $1-\frac{2}{3} W^{2}$ & 0 \\
3 & $3 \sqrt{2} Y$ & $A$ \\
4 & $1-\frac{4}{3} W^{2}+\frac{2}{9} W^{4}$ & 0 \\
5 & $\sqrt{2} Y\left(6-W^{2}\right)$ & $A\left(2-\frac{1}{3} W^{2}\right)$ \\
6 & (i) $1-2 W^{2}+\frac{2}{3} W^{4}-\frac{4}{81} W^{6}(\mathrm{ii}) \frac{1}{81} \sqrt{\frac{2}{5}}\left(2 W^{6}-729 Y^{2}\right)$ & $(3 / \sqrt{5}) A Y$ \\
7 & $(2 / 3 \sqrt{5})\left(45-15 W^{2}+W^{4}\right) Y$ & $\sqrt{10} A\left(1-\frac{1}{3} W^{2}+\frac{1}{45} W^{4}\right)$ \\
8 & (i) $1+\frac{2}{243} W^{2}\left(W^{2}-6\right)\left(54-18 W^{2}+W^{4}\right)$ & $3 \sqrt{\frac{7}{5}}\left(1-\frac{2}{21} W^{2}\right) A Y$ \\
& (ii) $\frac{1}{243} \sqrt{\frac{2}{35}}\left(2 W^{2}-21\right)\left(2 W^{6}-729 Y^{2}\right)$ & (i) $(A / \sqrt{5})\left(-10+5 W^{2}-\frac{2}{3} W^{4}+\frac{2}{81} W^{6}\right)$ \\
9 & (i) $\sqrt{\frac{6}{155}} Y\left(-30+15 W^{2}-2 W^{4}+18 Y^{2}\right)$ & (ii) $(A / 81 \sqrt{105})\left(W^{6}-729 Y^{2}\right)$ \\
\hline \hline
\end{tabular}

$$
\begin{aligned}
\left\langle p_{m^{\prime}}, p_{m}\right\rangle= & \int_{0}^{\infty} e^{-\frac{N-1}{N} W^{2}} W^{N-2} d W \\
& \times \int_{0}^{2 \pi} d \phi_{N-2} \prod_{k=1}^{N-3} \int_{0}^{\pi} \sin ^{N-2-k}\left(\phi_{k}\right) d \phi_{k} \\
& \times p_{m^{\prime}}\left(W, \phi_{1}, \ldots, \phi_{N-2}\right) p_{m}\left(W, \phi_{1}, \ldots, \phi_{N-2}\right),
\end{aligned}
$$

where the Jacobian determinant from Eq. (14) has already been explicitly included. One transforms the tuple $\left\{\bar{n}_{1}, \ldots, \bar{n}_{N}\right\}$ into $(N-1)$-dim spherical coordinates via the barycentric coordinates detailed in Appendix A.

The bosonic primitive basis is spanned by the elementary symmetric polynomials $\left\{S_{2}, S_{3}, \ldots, S_{N}\right\}=$ $\left\{\sum_{i j} \bar{n}_{i} \bar{n}_{j}, \sum_{i j k} \bar{n}_{i} \bar{n}_{j} \bar{n}_{k}, \ldots, \prod_{j} \bar{n}_{j}\right\} \quad$ and combinations thereof. For instance, with $N=5$ particles at degree $m=6$, there are three possible primitive polynomials: $S_{2}^{3}, S_{3}^{2}$, and $S_{2} S_{4}$. The fermionic primitive basis is spanned by all the symmetric polynomials as above, times the degree $\left(\begin{array}{c}N \\ 2\end{array}\right)$ Vandermonde determinant $\prod_{i<j}\left(\bar{n}_{i}-\bar{n}_{j}\right)$ shown in Fig. 4.

From the examples above, one easily deduces the degeneracy of the PPs $U_{m}^{N}$ to be $P(m, N)-P(m-1, N)$ for bosons and $P\left(m-\left(\begin{array}{c}N \\ 2\end{array}\right), N\right)-P\left(m-1-\left(\begin{array}{c}N \\ 2\end{array}\right), N\right)$ for fermions, where $P(m, N)$ is the number of partitions of the integer $N$ into at most $m$ parts $[42,43]$. In particular, the degeneracy is always nontrivial $(\geq 2)$ whenever $N \geq 4$ and $m \geq 4$.

\section{GEOMETRIC CONSTRUCTION OF PSEUDOPOTENTIALS: SPINFUL CASE}

In the presence of "internal degrees of freedom" (DOFs) which we also refer to as "spins" or "components" for simplicity, there are considerably more possibilities for the diverse forms of the PPs. This is because the PPs consist of products of spatial and spin parts, and either part can have
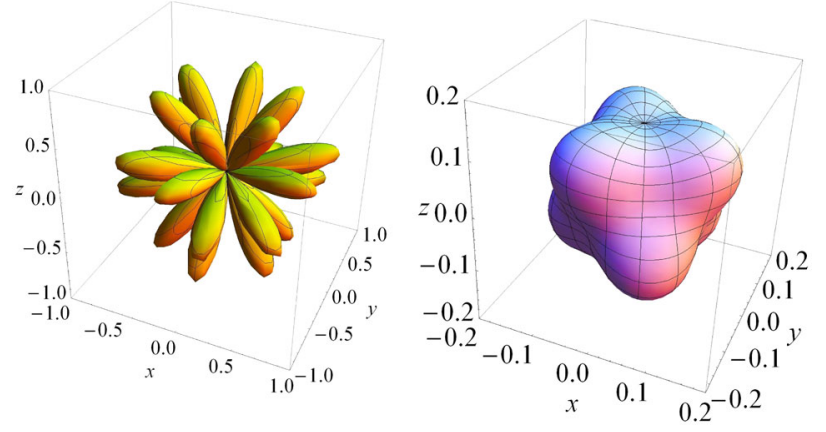

FIG. 4. The primitive polynomials have beautiful geometric shapes when plotted in $N-1$-dim spherical coordinates. Shown above are the constant $W$ plots of the antisymmetric polynomial $A=\left(\bar{n}_{1}-\bar{n}_{2}\right)\left(\bar{n}_{1}-\bar{n}_{3}\right)\left(\bar{n}_{1}-\bar{n}_{4}\right)\left(\bar{n}_{2}-\bar{n}_{3}\right)\left(\bar{n}_{2}-\bar{n}_{4}\right)\left(\bar{n}_{3}-\bar{n}_{4}\right) \quad$ (left diagram) and the degree $m=4$ symmetric expression in the orthonormalized space spanned by the elementary symmetric polynomials $1, S_{2}, S_{3}, S_{4}$, and $S_{2}^{2}$ (right diagram). On the left, there are 24 lobes that each maximally avoid the vertices of the tetrahedron (3-simplex). On the right, the six lobes lie around the centers of the six edges of the tetrahedron, where two of the $\bar{n}_{i}$ 's are equal. 
many possible symmetry types, as long as they conspire to produce an overall (anti)symmetric PP in the case of (fermions) bosons.

A generic multicomponent PP takes the form

$$
U_{m}^{N}=\sum_{\lambda} \sum_{\sigma} U_{m, \lambda}^{N}\left|\sigma^{\lambda}\right\rangle\left\langle\sigma^{\lambda}\right|
$$

The notation here requires some explanation. $\lambda=$ $\left[\lambda_{1}, \lambda_{2}, \ldots\right]$ defines a partition of all particles into several subgroups $\lambda_{i}$, each of which imposes a symmetry constraint among particles of this subgroup. Associated with this partition, or symmetry type, is $U_{m, \lambda}^{N}$, which is an $m$ th order spatial term [in $\left(\bar{n}_{1}, \ldots, \bar{n}_{N}\right)$ space] exhibiting this symmetry. $\left|\sigma^{\lambda}\right\rangle=\left|\sigma_{1} \sigma_{2} \ldots\right\rangle$ refers to an (internal) spin basis that is consistent with the symmetry type $\lambda$. Each symmetry type corresponds to a partition of $N$, with $\sum_{j} \lambda_{j}=N$. For $N$ bosons, $\lambda$ represents the situation where there is permutation symmetry among the first $\lambda_{1}$ particles and the next $\lambda_{2}$ particles, etc., but no additional symmetry between the $\lambda_{i}$ subsets. This is often represented by the Young tableau with $\lambda_{i}$ boxes in the $i$ th row. For fermions, we use the conjugate representation $\bar{\lambda}$, with the rows replaced by columns and symmetry conditions replaced by antisymmetry ones. For instance, the totally (anti)symmetric types are $[1,1, \ldots, 1]$ and $[N]$, respectively.

Hence, the space of PPs is specified by three parameters: $N$, the number of particles interacting with each other; $m$, the total relative angular momentum or the total polynomial degree in $\bar{n}_{i}$; and $b$, the number of internal DOFs (spin). While the symmetry type $\lambda$, and hence $U_{m, \lambda}^{N}$, depends only on $N$ and $m$, the set of possible $\left|\sigma^{\lambda}\right\rangle$ also depends on $b$. To further illustrate our notation, we specify $N, m, b$ parameters for the interactions relevant to some commonly known states: In the archetypical single-layer FQH states, we have $b=1 \mathrm{com}$ ponents, and the $N=2$ PP interactions for the Laughlin state penalize pairs of particles with relative angular momentum $m$, where $\nu=1 /(m+2)$ is the filling fraction. For bilayer FQH states, we have $b=2$ and $N=2$-body interactions. PPs as energy penalties in the [2] sector with angular momentum $<m$ and $[1,1]$ sectors with angular momentum $<n$ give rise to the Halperin $(m m n)$ states. Here, the [2] sector is also known as the triplet channel, as it is spanned by the following three basis vectors: $\{|\uparrow \uparrow\rangle,|\uparrow \downarrow\rangle+$ $|\downarrow \uparrow\rangle,|\downarrow \downarrow\rangle\}$. By contrast, the [1,1] sector only contains $\{|\uparrow \downarrow\rangle-|\downarrow \uparrow\rangle\}$, as dictated by antisymmetry.

\section{A. Multicomponent pseudopotentials for a given symmetry}

The construction of multicomponent PPs here parallels that of multicomponent wave functions described in Ref. [43]. For completeness, we first review this construction and proceed to show how an orthonormal multicomponent PP basis, adapted to the cylinder or torus, can be explicitly found through the geometric approach. We describe how to first find the spatial part of the PP $U_{m, \lambda}^{N}$, and second the spin basis $\left|\sigma^{\lambda}\right\rangle$.

\section{Spatial part}

For each symmetry type $\lambda$, we can construct the spatial part $U_{m, \lambda}^{N}$ with elementary symmetric polynomials in subsets of the particle indices $\bar{n}_{i}=n_{i}-n / N$. They are, for instance, $\quad S_{1,12}=\bar{n}_{1}+\bar{n}_{2}, \quad S_{2,234}=\bar{n}_{2} \bar{n}_{3}+\bar{n}_{3} \bar{n}_{4}+$ $\bar{n}_{2} \bar{n}_{4}$, etc. Of course, we must have $S_{1,123 \ldots N}=\sum_{i} \bar{n}_{i}=0$.

Like in the single-component case, the spatial part $U_{m, \lambda}^{N}$ consists of a primitive polynomial that enforces the symmetry and a totally symmetric factor that does not change the symmetry. Here, the main step in the multicomponent generalization is the replacement of primitive polynomials 1 and $A$ by primitive polynomials consistent with the symmetry type $\lambda$. As the simplest example, the primitive polynomial in $U_{m=1,[2,1]}^{N=3}$ is $S_{1,12}=-\bar{n}_{3}$ (and cyclic permutations). It is the only possible degree $m=1$ expression symmetric in two (but not all three) of the indices.

In general, there can be more than one candidate monomial obeying a symmetry consistent with $\lambda$. For instance, for $U_{m=2,[2,1]}^{N=3}$ they are $S_{1,12}^{2}=\left(\bar{n}_{1}+\bar{n}_{2}\right)^{2}$ and $S_{2,12}=\bar{n}_{1} \bar{n}_{2}$ (and cyclic permutations thereof). To find the primitive polynomials, we have to construct one or more linear combinations of these terms, which do not have any higher symmetry other than [2,1] (i.e., in this case, this higher-symmetry channel could be [3]). Elementary computation reveals that the only primitive polynomial should be $S_{1,12}^{2}+2 S_{2,12}$ because it is the only linear combination that is manifestly symmetric in indices 1,2 and disappears upon symmetrization over all three particles.

$N=4, m=2, \lambda=[2,2]$. - As a more involved example, we demonstrate how to find the primitive polynomial corresponding to the symmetry type [2,2]. Independent monomials that satisfy this symmetry include $S_{1,12}^{2}, S_{2,12}$, and $S_{2,34}$. The primitive polynomial is then given by the linear combination

$$
S_{1,12}^{2}+\mu_{1} S_{2,12}+\mu_{2} S_{2,34},
$$

with $\mu_{1}$ and $\mu_{2}$ to be determined by demanding that the linear combination disappears upon symmetrizing over permutations under [3,1] and [4]. The symmetrized sums are

$$
\begin{aligned}
& 2\left[\left(n_{2}+n_{3}\right)^{2}+\left(n_{2}+n_{4}\right)^{2}+\left(n_{3}+n_{4}\right)^{2}\right. \\
& \left.\quad+\mu_{1}\left(n_{1} n_{2}+n_{1} n_{3}+n_{2} n_{3}\right)+\mu_{2}\left(n_{1} n_{4}+n_{2} n_{4}+n_{3} n_{4}\right)\right]
\end{aligned}
$$

and 
$4\left(n_{2}^{2}+n_{3}^{2}+n_{4}^{2}+n_{2} n_{3}+n_{3} n_{4}+n_{2} n_{4}\right)\left(4-\mu_{1}-\mu_{2}\right)$.

Setting them both to zero, we find $\mu_{1}=\mu_{2}=2$, so the primitive polynomial is $S_{1,12}^{2}+2 S_{2,12}+2 S_{2,34}$.

$N=3, m=2, \lambda=[2,1]$. - The above procedure works for arbitrarily complicated cases but quickly becomes cumbersome. This is when our geometric approach again becomes useful. We first write down the relevant monomials in barycentric coordinates given by Eq. (22) for $N=3$ bodies [or Eq. (A12) for general $N$ ]. The coefficients in the primitive polynomial can then be elegantly determined through graphical inspection. We demonstrate this explicitly by revisiting the example on $\lambda=[2,1]$. Recall that the primitive polynomial (call it $\beta_{m=2} \equiv \beta_{2}$ ) is a linear combination of $S_{1,12}^{2}$ and $S_{2,12}$, i.e.,

$$
\begin{aligned}
\beta_{2} & =S_{1,12}^{2}+\mu S_{2,12} \\
& =-\frac{W^{2}}{9 \kappa}(\mu-2+(1+\mu)(\cos 2 \theta-\sqrt{3} \sin 2 \theta)),
\end{aligned}
$$

where $\theta=0,2 \pi / 3,4 \pi / 3$ points towards the vertices favoring $\bar{n}_{1}, \bar{n}_{2}, \bar{n}_{3}$, respectively. The correct value of $\mu$ will cause $\beta_{2}$ to disappear under symmetrization of the three particles. Graphically, this means that the lobes of three copies of the plot of $\beta_{2}$, each rotated an angle $2 \pi / 3$ from each other, must cancel upon addition. This is illustrated in Fig. 5, where $\mu=2$ is readily identified as the correct value.

All in all, we have the $m=2$ primitive polynomial for $[2,1]$ :

$\beta_{2}=\left(\bar{n}_{3}\right)^{2}+2 \bar{n}_{1} \bar{n}_{2}=\frac{W^{2}}{3}(-\cos 2 \theta+\sqrt{3} \sin 2 \theta)$.

$N=3, m=1, \lambda=[2,1]$. - The reader is invited to also visualize the simpler case for the $[2,1]$ sector, which takes the form

$$
\beta_{1}=S_{1,12}=-\bar{n}_{3}=\frac{W}{3 \kappa}(\cos \theta+\sqrt{3} \sin \theta) .
$$

Compared to the antisymmetric case for fermions, we have two primitive polynomials $\beta_{1}, \beta_{2}$, instead of just $A$. The primitive polynomials for a wide variety of cases are also listed in Tables II and III of Ref. [43], and can alternatively be systematically derived via the theory of Matric units explained in the appendix of the same reference.

\section{Admissible spin bases}

In general, not all possible $N$-particle spin bases survive under symmetrization with respect to a given symmetry type $\lambda$. Only those that survive should be included, i.e., are admissible, in the set of basis in $\left|\sigma^{\lambda}\right\rangle$. For instance, the basis

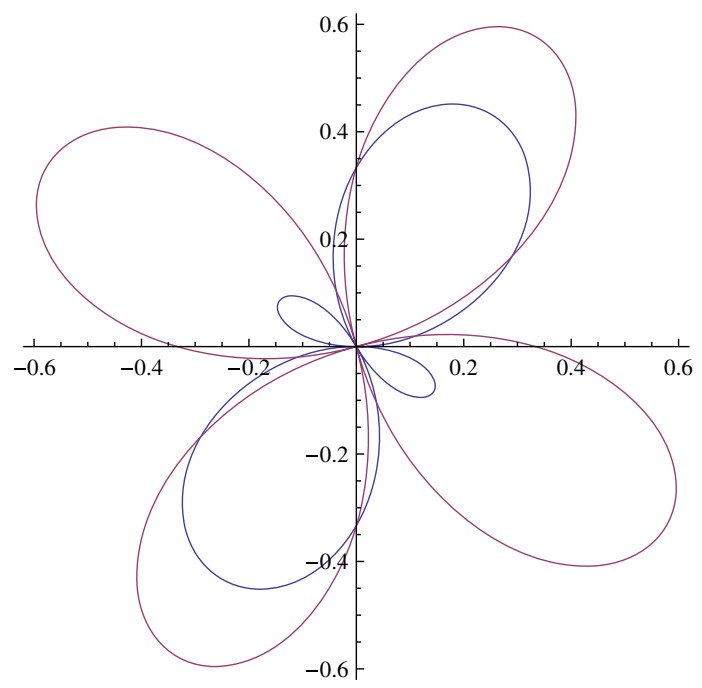

FIG. 5. Polar plots of $\beta_{2}(\theta)$ as a function of $\theta$, with $\mu=0.5$ (blue curve) and $\mu=2$ (purple curve). $\beta_{2}(\theta)+\beta_{2}(\theta+2 \pi / 3)+$ $\beta_{2}(\theta+4 \pi / 3)$ is zero only when the lobes are of equal size, which is the case for $\mu=2$ only.

$|\alpha \alpha \alpha\rangle$ does not appear in $\left|\sigma^{[2,1]}\right\rangle$. To see this, try symmetrizing the combination $p\left(\bar{n}_{1}, \bar{n}_{2}, \bar{n}_{3}\right)|\alpha \alpha \alpha\rangle$ subject to the condition that $p\left(\bar{n}_{1}, \bar{n}_{2}, \bar{n}_{3}\right)$ has no higher symmetry than $\lambda=[2,1]$, i.e., the totally symmetrized sum $\mathcal{S}\left[p\left(\bar{n}_{1}, \bar{n}_{2}, \bar{n}_{3}\right)\right]=0$. Obviously, $p\left(\bar{n}_{1}, \bar{n}_{2}, \bar{n}_{3}\right)|\alpha \alpha \alpha\rangle$ then has to symmetrize to zero and should not be included in $[2,1]$.

We can write down the set of admissible bases by looking at the labelings of semistandard Young tableaux. From Ref. [43], the admissible bases in $\left|\sigma^{\lambda}\right\rangle$, up to permutations, are in one-to-one correspondence with the labelings of semistandard Young tableaux with numbers 1 to $N$. (A semistandard Young tableau has nondecreasing entries along each row and strictly increasing entries along each column.) For instance, a symmetry type of $[3,1]$ with $b=3$ corresponds to labelings (with labels $a, b, c$ defined to be in increasing order):

$[a a a, b],[a a b, b],[a a c, b],[a b b, b],[a b c, b]$, $[a c c, b],[a a a, c],[a a b, c],[a a c, c],[a b b, c]$, $[a b c, c],[a c c, c],[b b b, c],[b b c, c],[b c c, c]$ and $[c c c, c]$,

where rows are separated by commas. From these, the admissible states for $\lambda=[3,1]$ can be written down by copying the labelings verbatim:

$$
\begin{aligned}
& |a a a b\rangle,|a a b b\rangle,|a a c b\rangle,|a b b b\rangle,|a b c b\rangle, \\
& |a c c b\rangle,|a a a c\rangle,|a a b c\rangle,|a a c c\rangle,|a b b c\rangle, \\
& |a b c c\rangle,|a c c c\rangle,|b b b c\rangle,|b b c c\rangle,|b c c c\rangle, \quad \text { and }|c c c c\rangle .
\end{aligned}
$$


TABLE IV. Summary of the PP construction procedure for the multicomponent case. Examples of this procedure are given in Sec. V.

(1) Given $N, m$, and $b$, specify which symmetry type the PP is associated with. For example, we specify the [2] or the [1,1] channel when computing PPs realizing the fermionic Halperin states in bilayer $\mathrm{QH}$ systems $(b=2, N=2)$.

(2) Next, determine the appropriate primitive polynomials by finding the coefficients multiplying the allowed monomials. Then, multiply the primitive polynomial by symmetric polynomials and orthogonalize to obtain the spatial parts $U_{m, \lambda}^{N}$.

(3) Finally, choose the desired admissible spin channels and (anti)symmetrize the resultant product of the spatial and spin parts depending on whether we want a bosonic or fermionic PP.

In the Young tableau corresponding to $\lambda$, each row represents a set of particles that are symmetric with each other in the $\lambda$ representation. The requirement that labels increase monotonically within each row defines an ordering and prevents the repeated listing of basis states related by permutation. The requirement of strictly increasing labelings down each column also prevents this and also avoids the listing of bases that do not survive under symmetry constraints.

With these preliminary considerations, we are now in a position to formulate the general recipe for constructing PPs in the multicomponent case, which is given in Table IV. In the following section, we apply this recipe to the case of $\mathrm{SU}(2)$ spins with three-body interactions.

\section{B. Example: $N=3$-body case for $\mathrm{SU}(2)$ spins}

Here, we explicitly work out the multicomponent case $b=2$, which is also the most common multicomponent scenario. We focus on $N=3$-body interactions to illustrate the nontrivial aspects of our approach.

The symmetry type is given by $\left[\lambda_{1}, \lambda_{2}\right]=$ $[N / 2+S, N / 2-S]$, where $S$ is the total spin of the particles. Let us denote the spins by $\uparrow, \downarrow$. There are only $N=3$ boxes in the Young tableaux, with the following possible symmetry types: [3], [2,1], [1,1,1].

For type $\lambda=[3]$, the possible spin bases are $|\uparrow \uparrow \uparrow\rangle$, $|\uparrow \uparrow \downarrow\rangle,|\uparrow \downarrow \downarrow\rangle$, and $|\downarrow \downarrow \downarrow\rangle$. For $\lambda=[2,1]$, the possible spin bases are $|\uparrow \downarrow \downarrow\rangle,|\uparrow \uparrow \downarrow\rangle$, corresponding to tableau labelings $[\uparrow \downarrow, \downarrow]$ and $[\uparrow \uparrow, \downarrow]$, respectively. For $\lambda=[1,1,1]$, there is actually no admissible spin basis: Total (internal DOF) antisymmetry is impossible for three particles, if there are only two different spin states to choose from.

Consider bosonic particles in the following. The symmetry type [3] case corresponds to the primitive polynomial 1, so the resultant PP takes the same form as in the singlecomponent case. After symmetrizing the spin part, we have the following available sets of spin channels for [3]:

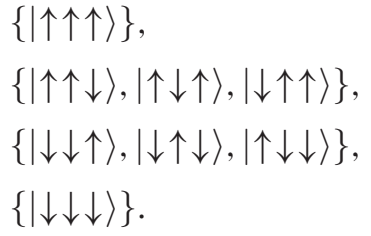

For the PP $U_{m,[3]}^{3} \propto \kappa^{3} \sum_{n} b_{n}^{m \dagger} b_{n}^{m}$, we have

$$
b_{n}^{m \dagger}|0\rangle=\sum_{\sum_{j} n_{j}=n} p_{m} e^{-\frac{1}{3} W^{2}}|\uparrow \uparrow \uparrow\rangle
$$

for $S=3 / 2$, and

$$
b_{n}^{\dagger m}|0\rangle=\mathcal{S}\left[\sum_{\sum_{j} n_{j}=n} p_{m} e^{-\frac{1}{3} W^{2}}|\uparrow \uparrow \downarrow\rangle\right]
$$

for $S=1 / 2$. Other contributions with $S \rightarrow-S$ can be obtained via the identification $|\uparrow\rangle \leftrightarrow|\downarrow\rangle$. Here, $|\uparrow \uparrow \downarrow\rangle$ is the shorthand for $c_{n_{1} \uparrow}^{\dagger} c_{n_{2} \uparrow}^{\dagger} c_{n_{3} \downarrow}^{\dagger}|0\rangle$, etc., and $p_{m} \equiv p_{m,[3]}$ refers to the same polynomial as in the single-component case.

To construct the orthonormal PP basis for the first few $m$, we orthogonalize the set $\left\{\beta_{1}, \beta_{2}, \beta_{1} W^{2}, \beta_{2} W^{2}, \beta_{1} Y, \beta_{2} Y\right.$, $\left.\beta_{1} W^{4}, \ldots\right\}$. The results are shown in Table V. Notice that the first PP for symmetry type [2,1] occurs at $m=1$, whereas that of single-component bosons or fermions occurs at $m=0$ and $m=3$, respectively. Indeed, there are more ways of constructing PP polynomials when only a subgroup of the full symmetric or alternating group is involved. The onset of PPs degenerate in $m$ also occurs earlier, at $m=4$.

TABLE V. The polynomials $p_{m}$ for the first few $N=3$-body PPs for bosons in the total spin $|S|=\frac{1}{2}$, i.e., $\lambda=[2,1]$ channel. The primitive polynomials $\beta_{1}$ and $\beta_{2}$ are given by Eqs. (33) and (34). The $\cos 4 \theta, \sin 4 \theta, \cos 5 \theta, \sin 5 \theta$ can all be decomposed into the elementary symmetric polynomials and primitive polynomials $W, Y, \beta_{1}$, and $\beta_{2}$.

\begin{tabular}{ll}
\hline \hline$m$ & \multicolumn{1}{c}{$p_{m,[2,1]}\left(\beta_{1}, \beta_{2}, W, Y\right)$} \\
\hline 1 & $\beta_{1}$ \\
2 & $(1 / \sqrt{3}) \beta_{2}$ \\
3 & $\frac{1}{3} \sqrt{2} \beta_{1}\left(-3+W^{2}\right)$ \\
$4(\mathrm{i})$ & $(1 / \sqrt{5})\left(\beta_{2}+6 \beta_{1} Y\right)$ \\
$4(\mathrm{ii})$ & $(1 / 27 \sqrt{5})\left[-54 \beta_{2}+12 \beta_{2} W^{2}+W^{4}(\cos [4 \theta]\right.$ \\
& $\quad+\sqrt{3} \sin [4 \theta])]$ \\
5 (i) & $(1 / \sqrt{33})\left[\beta_{1}\left(-3+2 W^{2}\right)+6 \beta_{2} Y\right]$ \\
5 (ii) & $\frac{1}{81} \sqrt{\frac{2}{55}}\left[-\sqrt{3} W^{5} \cos [5 \theta]+3\left(5 \sqrt{3} \beta_{1}\left(27-18 W^{2}+2 W^{4}\right)\right.\right.$ \\
& $\left.\left.\quad+W^{5} \sin [5 \theta]\right)\right]$ \\
\hline \hline
\end{tabular}


To illustrate the full procedure for PP construction with internal DOFs, we detail the case of $S=-1 / 2$ below. For $m=1$,

$$
\begin{aligned}
b_{n}^{1 \dagger}|0\rangle= & \mathcal{S}\left[\sum_{\sum_{j} n_{j}=n}\left(-\bar{n}_{3}\right) e^{-\frac{1}{3} W^{2}}|\uparrow \downarrow \downarrow\rangle\right] \\
= & -e^{-\frac{1}{3} W^{2}}\left[\left(\bar{n}_{2}+\bar{n}_{3}\right)|\uparrow \downarrow \downarrow\rangle\right. \\
& \left.+\left(\bar{n}_{1}+\bar{n}_{3}\right)|\downarrow \uparrow \downarrow\rangle+\left(\bar{n}_{1}+\bar{n}_{2}\right)|\downarrow \downarrow \uparrow\rangle\right] \\
= & e^{-\frac{1}{3} W^{2}}\left[\bar{n}_{1}|\uparrow \downarrow \downarrow\rangle+\bar{n}_{2}|\downarrow \uparrow \downarrow\rangle+\bar{n}_{3}|\downarrow \downarrow \uparrow\rangle\right],
\end{aligned}
$$

while for $m=2$, we have

$$
\begin{aligned}
b_{n}^{2 \dagger}|0\rangle= & \mathcal{S}\left[\sum_{\sum_{j} n_{j}=n}\left(\left(\bar{n}_{3}\right)^{2}+2 \bar{n}_{1} \bar{n}_{2}\right) e^{-\frac{1}{3} W^{2}}|\uparrow \downarrow \downarrow\rangle\right] \\
= & e^{-\frac{1}{3} W^{2}}\left[\left(\left(\bar{n}_{2}\right)^{2}+\left(\bar{n}_{3}\right)^{2}+2 \bar{n}_{1}\left(\bar{n}_{2}+\bar{n}_{3}\right)\right)|\uparrow \downarrow \downarrow\rangle\right. \\
& +\left(\left(\bar{n}_{1}\right)^{2}+\left(\bar{n}_{2}\right)^{2}+2 \bar{n}_{3}\left(\bar{n}_{1}+\bar{n}_{2}\right)\right)|\downarrow \downarrow \uparrow\rangle \\
& \left.+\left(\left(\bar{n}_{1}\right)^{2}+\left(\bar{n}_{3}\right)^{2}+2 \bar{n}_{2}\left(\bar{n}_{1}+\bar{n}_{3}\right)\right)|\downarrow \uparrow \downarrow\rangle\right] .
\end{aligned}
$$

Expressions for $S=1 / 2$ are obtained via $|\uparrow\rangle \leftrightarrow|\downarrow\rangle$.

For the present case of $\mathrm{SU}(2)$ spins, i.e., $b=2$, there exists a nice closed-form generating function for the dimension of the spatial basis for each $m$. Define the generating function $Z_{\left[\lambda_{1}, \lambda_{2}\right]}(q)=\sum_{m} d\left(\left[\lambda_{1}, \lambda_{2}\right] ; m\right) q^{m}$, where $d\left(\left[\lambda_{1}, \lambda_{2}\right] ; m\right)$ is the number of different polynomials with degree $m$ and symmetry type $\left[\lambda_{1}, \lambda_{2}\right]$. It can be shown that for bosons,

$$
\begin{aligned}
Z_{\left[\lambda_{1}, \lambda_{2}\right]}(q)= & \frac{1-q}{\prod_{m=1}^{\lambda_{1}}\left(1-q^{m}\right) \prod_{n=1}^{\lambda_{2}}\left(1-q^{n}\right)} \\
& -\frac{1-q}{\prod_{m=1}^{\lambda_{1}+1}\left(1-q^{m}\right) \prod_{n=1}^{\lambda_{2}-1}\left(1-q^{n}\right)} .
\end{aligned}
$$

This result is obtained [43] by considering the dimensionality from two symmetry subsets and then subtracting overlaps from the higher-symmetry case $\left[\lambda_{1}+1, \lambda_{2}-1\right]$. The dimension is related to the $q$-binomial coefficient. For $b>2$, however, the situation is much more complicated, involving Kostka coefficients that count the number of semisimple labelings of $\lambda$ with a given alphabet.

\section{PSEUDOPOTENTIAL HAMILTONIANS: CASE STUDIES}

In this section, we provide several applications of the general pseudopotential construction developed in the previous sections, with examples arranged in the order of increasing complexity. We start with spin-polarized states (Sec. VA), whose Hamiltonians have been obtained via alternative methods and are well known in the literature.
As an illustration of the method, we provide a detailed derivation of the fermionic Gaffnian parent Hamiltonian. Note that the resulting second-quantized form of the Hamiltonian applies (with minimal modifications) to both cylinder and torus geometries. In addition to pedagogical examples that illustrate our approach, we construct pseudopotential Hamiltonians for some non-Abelian states that have not been available in the literature for any geometry.

The main sequence of steps is stated as follows: (i) Assuming that the ground-state wave function is known in the first-quantized form, find its thin-torus pattern (this step has been frequently discussed in the literature; for completeness, we provide a brief summary in Appendix C). (ii) Using the formalism developed in the previous sections, write down the parent Hamiltonian corresponding to the root pattern in the second-quantized form. (iii) Use numerics (exact diagonalization) to verify that the ground state of the proposed Hamiltonian is indeed given by the initial wave function. The verification criteria include testing for the unique zero-energy ground state at the given filling factor, the correct thin-cylinder root patterns as the circumference of the cylinder is taken to zero, and the level counting of entanglement spectra. For the purpose of numerical calculations, we place a finite total number of particles $N$ on the surface of a torus or an open cylinder. The two linear dimensions of the Hall surface ( $L$ and $H$ ) satisfy the relation $L H=2 \pi l_{B}^{2} N_{\text {orb}}$, where $N_{\text {orb }}$ is the number of available orbitals (it is equal to $N_{\phi}$ on the torus and equal to $N_{\phi}+1$ on the cylinder). Unless stated otherwise, we also assume $L=H$.

\section{A. Spin-polarized states}

The construction of two-body as well as the shortestrange three-body pseudopotential Hamiltonians has been discussed in depth in the literature (see, e.g., Refs. [4,911]). Starting from there, as our most elementary example for spin-polarized particles, we consider ground states of longer-ranged three-body potentials: the fermionic Gaffnian state at filling $\nu=2 / 5$ as well as the Haffnian and the generalized Moore-Read Pfaffian state at filling $\nu=1 / q$, for which the $q=4$ case will be discussed in detail.

\section{Fermionic Gaffnian}

The derivation of the fermionic Gaffnian parent Hamiltonian is summarized in Fig. 6. The wave function of the Gaffnian state on the infinite plane is given by [54]

$$
\Psi_{\mathrm{Gaf}}=\mathcal{A}\left\{\Psi_{332}\left(\left\{z_{\uparrow}\right\},\left\{z_{\downarrow}\right\}\right) \operatorname{Per}\left(\frac{1}{z_{\uparrow}-z_{\downarrow}}\right)\right\},
$$

where we have suppressed the usual Gaussian factors. Although physically a one-component (spin-polarized) 
(1) First quantized wave function:

(4) Energy spectrum on the torus

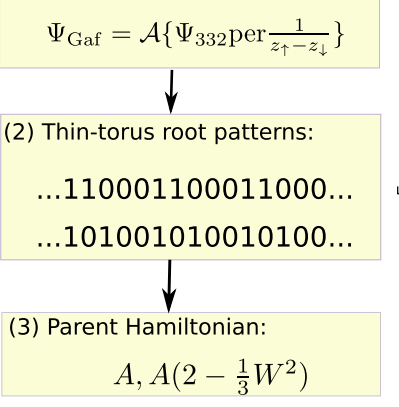

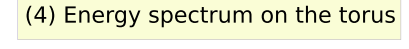

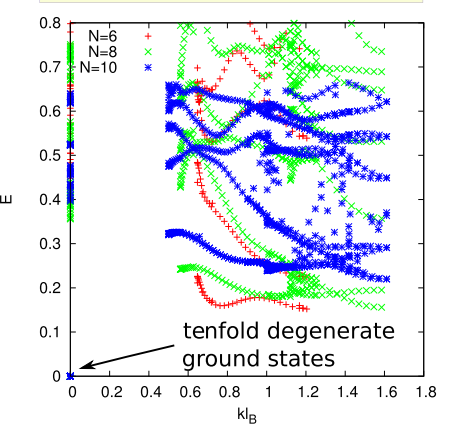

FIG. 6. Main steps in constructing the fermionic Gaffnian parent Hamiltonian. (i) Assume the first quantized wave function, in this example given by Eq. (40). (ii) Find the corresponding thin-torus root patterns (here, $1100011000 \ldots$ and $1010010100 \ldots$... (iii) Construct the parent Hamiltonian by consulting Eq. (26) and Table III. (iv) Verify the construction and compute properties by exact diagonalization. Here, this is performed on a hexagonal torus with $N=6,8,10$ particles, finding a tenfold ground state with exactly zero energy. The energy scale is set by $\gamma=1$ in Eq. (43). As the torus is stretched along one direction, the ground states evolve to the expected patterns $1100011000 \ldots$ and $1010010100 \ldots$.. In addition, we obtain the entire low-lying neutral excitation spectrum, allowing one, in principle, to extract many other properties of the system.

state, the above wave function reflects the underlying "twocomponent" nature of the Gaffnian state: In order to write down the wave function, we have divided electrons into two groups, $\uparrow$ and $\downarrow$ (not to be confused with physical spin), that are correlated through the Jastrow factors within the 332 Halperin state, which is defined as

$$
\begin{aligned}
\Psi_{332}\left(\left\{z_{\uparrow}, z_{\downarrow}\right\}\right)= & \prod_{i<j}\left(z_{\uparrow, i}-z_{\uparrow, j}\right)^{3} \prod_{i<j}\left(z_{\downarrow, i}-z_{\downarrow, j}\right)^{3} \\
& \times \prod_{i, j}\left(z_{\uparrow, i}-z_{\downarrow, j}\right)^{2},
\end{aligned}
$$

as well as the "permanent"

$$
\operatorname{Per}\left(\frac{1}{z_{\uparrow}-z_{\downarrow}}\right)=\sum_{\sigma} \frac{1}{\prod_{j}\left(z_{\uparrow j}-z_{\downarrow \sigma(j)}\right)} .
$$

Ultimately, the distinction between $\uparrow$ and $\downarrow$ particles is erased by the overall antisymmetrization $\mathcal{A}$, producing a well-defined single-component wave function.

Having obtained the first quantized wave function for the Gaffnian, the second step is to determine its thin cylinder root patterns. (Note that a similar but different analysis can also be executed on the sphere by analyzing the root partitions of the parent Hamiltonian null space [55]). The detailed procedure for finding the root patterns of Halperin bilayer states was given in Ref. [56], of which a brief summary is outlined in Appendix C. The Gaffnian wave function vanishes as power 6 as three particles are brought

together; hence, its thin-torus root patterns are $1100011000 \ldots$ and $1010010100 \ldots$, accordingly.

From the form of the root patterns, we conclude that we need $U_{m=3}^{N=3}$ and $U_{m=5}^{N=3}$ terms in order to build the parent Hamiltonian. The polynomial amplitudes for these terms can be found in Eq. (26) and Table III. The Gaffnian parent Hamiltonian therefore reads

$$
\begin{gathered}
H=\sum_{n} b_{n}^{\dagger} b_{n}+\gamma \sum_{n} d_{n}^{\dagger} d_{n}, \\
b_{n}=\sum_{\bar{n}_{1}+\bar{n}_{2}+\bar{n}_{3}=0} A\left(\bar{n}_{1}, \bar{n}_{2}, \bar{n}_{3}\right) e^{-\left(\kappa^{2} / 2\right) \sum_{j=1}^{3} \bar{n}_{j}^{2}} c_{n_{1}} c_{n_{2}} c_{n_{3}}, \\
d_{n}=\sum_{\bar{n}_{1}+\bar{n}_{2}+\bar{n}_{3}=0} A\left(\bar{n}_{1}, \bar{n}_{2}, \bar{n}_{3}\right)\left[2-\frac{1}{3} W^{2}\left(\bar{n}_{1}, \bar{n}_{2}, \bar{n}_{3}\right)\right] \\
\times e^{-\left(\kappa^{2} / 2\right) \sum_{j=1}^{3} \bar{n}_{j}^{2}} c_{n_{1}} c_{n_{2}} c_{n_{3}},
\end{gathered}
$$

where as before $\bar{n}_{j}=n_{j}-n / 3$. The Hamiltonian (43) assigns positive energies to $m=3,5$ in a cluster of three particles in an infinite system, while all the other energies are exactly zero. The constant $\gamma$ tunes the ratio of the two nonzero energies and can be set to any positive number. The precise value of $\gamma$ does not affect the ground-state wave function or its energy, but it does have an effect on the energetics of the low-lying excited states. In the following, for the sake of brevity, we refer to the Hamiltonian in Eq. (43) simply by $A+\gamma A\left(2-\frac{1}{3} W^{2}\right)$.

Finally, having obtained the parent Hamiltonian, we can verify that it yields the correct ground state we started from [Eq. (40)]. As we see in Fig. 6, exact diagonalization on the torus for $N=6,8,10$ electrons consistently finds a zeroenergy ground state that is tenfold degenerate and has zero momentum (i.e., does not break translation symmetry). One can further verify that we have obtained the correct ground state by computing its entanglement properties, as we discuss below. Another nontrivial check is to perform diagonalization on a torus stretched along one axis and directly identify the root patterns in the thin-torus limit. In doing so, we find, as expected, the ground states to evolve to the single Fock states $1100011000 \ldots$ and $1010010100 \ldots$ (and those obtained from these by COM translation), in agreement with the fermionic Gaffnian state.

\section{Pfaffians and Haffnians}

We next consider the generalized non-Abelian Pfaffian state at $1 / q$ filling $[8,11]$. The wave function in the disk geometry reads

$$
\operatorname{Pf}\left(\frac{1}{z_{i}-z_{j}}\right) \prod_{i<j}\left(z_{i}-z_{j}\right)^{q},
$$

where even (odd) $q$ corresponds to a fermionic (bosonic) state. The Pfaffian is defined as 


$$
\operatorname{Pf}(A)=\frac{1}{2^{n / 2}(n / 2) !} \sum_{\sigma \in S_{n}} \operatorname{sgn}(\sigma) \prod_{i=1}^{n / 2} A_{\sigma(2 i-1), \sigma(2 i)},
$$

where $A$ is the $n \times n$ skew-symmetric matrix $A_{i j}=1 /$ $\left(z_{i}-z_{j}\right)$ for $n$ even. The case $q=2$ reduces to the familiar Moore-Read state, which is the ground state of the purely three-body interaction $U_{3}^{3}$. One would naively expect that $q>2$ states can be obtained by adding two-body terms to $U_{3}^{3}$, but as one can explicitly verify, this is not the case.

By a power-counting procedure [11] (further elaborated on in Appendix C), the root configuration of the $1 / q$ Pfaffian state is given by

$$
10^{q-2} 10^{q} 10^{q-2} 10^{q} \ldots \ldots 10^{q-2} 10^{q} \ldots,
$$

where $0^{q}$ represents a string of $q$ zeros. It follows that its parent Hamiltonian is given by the three-body PP

$$
U_{m=3(q-1)}^{3},
$$

as well as all nonzero spinless two-body PPs

$$
U_{m<q-2}^{2} .
$$

For the more interesting $q=4$ case that we have studied numerically, we require the two-body $U_{1}^{2} \mathrm{PP}$ and threebody $U_{9, \text { (i) }}^{3}$ and $U_{9, \text { (ii) }}^{3}$ PPs given by Eq. (26) and Table III. [Again, we emphasize that $U$ 's promoted to how they appear in the Hamiltonian should be understood as polynomial amplitudes that enter the definition of operators $b_{n}^{\dagger}, b_{n}$ in Eq. (10).]

Note that labels (i),(ii) stand for two linearly independent three-body PPs that occur for $m=9$. Thus, the $1 / 4$ Pfaffian state is an example whose parent Hamiltonian contains a degenerate subspace of PPs. The state can alternatively be obtained numerically through Jack polynomials [57] and the root configuration given above. We have confirmed that the overlap between the ground state of the parent Hamiltonian and the Jack polynomial wave function is equal to 1 (within machine precision) for $q=4$ and $N=$ 6,8 electrons. Note that experiments [58] find some evidence for an incompressible, potentially non-Abelian, $\nu=1 / 4$ state. This may be attributed to the Pfaffian $1 / 4$ state, although theoretical calculations suggest that the Halperin 553 state is also a candidate [59].

For our final spin-polarized example, we consider the fermionic Haffnian state [60], whose bosonic counterpart was recently considered in Ref. [39]. The fermionic Haffnian occurs at the filling fraction $\nu=1 / 3$, and, up to COM translation, has the following root patterns [61] on the sphere: $110000110000 \ldots$ and $100100100100 \ldots$ The latter pattern is identical to that of the Laughlin state. The fermionic Haffnian vanishes as power 7 as three particles are brought together; hence, we need to impose an energy penalty for $m=6$ too, and the parent Hamiltonian consists of

$$
U_{3}^{3}, \quad U_{5}^{3}, \quad \text { and } \quad U_{6}^{3},
$$

which are given by Eq. (26) and Table III.

\section{B. Spinful states}

We have previously mentioned that if we allow the spin degree of freedom to enter, the number of possible states obviously becomes much richer with interactions involving three or more particles. A systematic investigation of these states is left for future work. Here, we content ourselves with illustrating our method by formulating parent Hamiltonians for several states that have been the subject of recent attention: the spinful Gaffnian [62] and the NASS states [63]. The Hamiltonians for these states have previously been written down for the sphere (or disk) geometry, which we now extend to the cylinder and torus. Furthermore, we propose the parent Hamiltonian for a certain type of state involving the permanent state ("221 times permanent" state [64]), for which the Hamiltonian was previously unknown.

In addition to formulating the parent Hamiltonians, we also computed the orbital entanglement spectrum (OES) and the particle entanglement spectrum (PES) for the respective states. Figure 7 illustrates the two types of partitioning in the case of an open cylinder. After performing the cut, the entanglement spectrum can still have some remaining symmetry that can be used to classify the Schmidt levels. For example, for the OES, the total number of particles $N_{A}$ and the total number of orbitals $l_{A}$ in the left subsystem remain good quantum numbers. On the other hand, for the PES, the translation or rotation symmetry of the full system is preserved. For an open cylinder, as in Fig. 7, this means that the total momentum $K_{y}^{A}$ of the left subsystem is also a good quantum number:

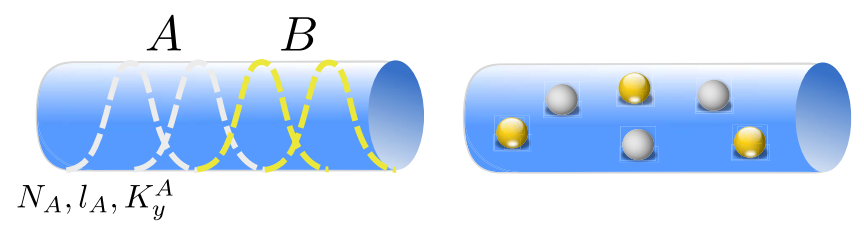

FIG. 7. Two choices of partitioning the system for computing the entanglement spectrum. The orbital cut (left diagram) amounts to partitioning the system into two groups of orbitals $A$ and $B$, and tracing out the orbitals in $B$ (marked yellow). The Schmidt levels of $A$ can be classified by the number of particles $N_{A}$, the number of orbitals $l_{A}$, and the total momentum $K_{y}^{A}$. An alternative choice is particle partitioning (right diagram), where some particles (marked in yellow) are traced out, regardless of their position. 


$$
K_{y}^{A}=\sum_{m \in A} m \hat{n}_{m}
$$

with $\hat{n}_{m}$ the density operator acting on the momentum orbital $m$. This linear momentum on the cylinder corresponds to the $L_{z}$ projection of angular momentum on the sphere. The PES on the sphere, however, is also invariant under full $\mathrm{SU}(2)$ rotation; i.e., it forms multiplets of $L^{2}$. This symmetry is absent on a cylinder, where we can only use $K_{y}^{A}$ to classify the levels of the entanglement spectrum. This is discussed in more detail in the example in Sec. VB 1. As we perform an orbital partition, the symmetry of the subsystem is reduced, and even on the sphere, the only remaining quantum number is $L_{z}$. Thus, the OES on the sphere can be directly compared with the OES on the open cylinder. Finally, for spinful states, the spin quantum number commutes with the reduced density operator of the OES and thus allows for additional resolution of the ES level counting [65].

\section{Spin-singlet Gaffnian state}

The spin-singlet Gaffnian state is a nontrivial spinful generalization of the bosonic spin-polarized Gaffnian state by Davenport et al. [62]. In addition to the two spinpolarized three-body terms with $S=3 / 2$, its parent Hamiltonian also contains the shortest-range $(m=1)$ term with $S=1 / 2$. In total, this encompasses the PPs

$$
U_{0, S=3 / 2}^{3}, \quad U_{2, S=3 / 2}^{3} \quad \text { and } \quad U_{1, S=1 / 2}^{3} .
$$

As such, the spin-singlet Gaffnian wave function vanishes as the third power in the $S=3 / 2$ channel and the second power in $S=1 / 2$. These projectors are given by Eqs. (26) and (35) and Table III for $U_{0,3 / 2}^{3}$ and $U_{2,3 / 2}^{3}$, and Eq. (37) for $U_{1, S=1 / 2}^{3}$ (with both spin orientations $|\uparrow\rangle \leftrightarrow|\downarrow\rangle$ ). In addition to these, we also add the total spin operator $S^{2}$ to our Hamiltonian, to ensure that the ground state is a spin singlet.

By diagonalizing the Hamiltonian (52) numerically, we find a zero-energy ground state at filling factor $\nu=4 / 5$ and shift of -3 on a finite cylinder for $N=4,8$, and 12 particles. We have furthermore computed the PES [27] for $N=12$ particles and $N_{\phi}=12$ flux quanta, shown in Fig. 8. As illustrated in Fig. 7 (right), to perform a PES "partition," we divide the system into parts $A$ and $B$, which both contain $N_{\phi}$ orbitals, but $N_{A}$ and $N_{B}$ particles, respectively, such that $N_{A}+N_{B}=N$. To obtain Fig. 8, we have traced out $N_{B}=6$ particles from the system. The PES obtained in this way provides information about the counting of quasihole excitations of the given state, as shown in Ref. [27].

We compare the counting in Fig. 8 with the corresponding PES obtained on the sphere in Davenport et al. [62]. Note that our result in Fig. 8 superficially looks different

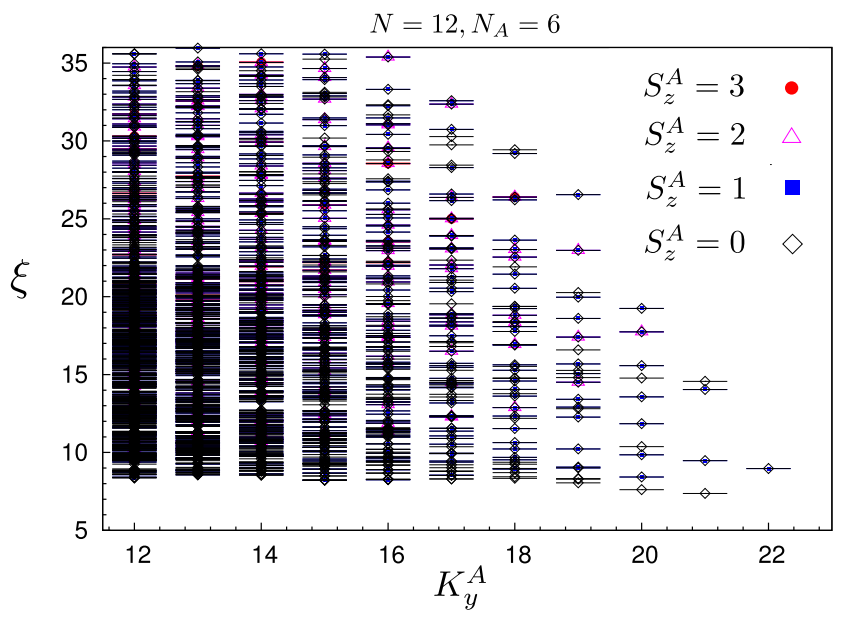

FIG. 8. PES of the spin-singlet Gaffnian state for $N=12$ particles and $N_{\phi}=12$ flux quanta on an open cylinder with aspect ratio equal to 1 . The spectrum is obtained by tracing out $N_{B}=N_{A}=6$ particles and plotted as a function of momentum $K_{y}^{A}$ in part $A$. Different values of $S_{z}^{A}$, the total $z$ component of spin in part $A$, are indicated in the inset.

from the result in Ref. [62]. This is because the PES partition preserves the symmetry of the ground state, causing the PES on the sphere to have exact rotational symmetry, which is absent for our open cylinder. In other words, on an open cylinder, the good quantum number after partitioning is only the linear momentum $K_{y}^{A}$. This quantum number, in turn, corresponds to the $L_{z}$ projection of angular momentum on the sphere. This, however, does not exhaust all symmetries of the PES on the sphere, where the full angular momentum $L^{2}$ is a good quantum number. This additional degeneracy of the PES is factored out in Ref. [62]. Furthermore, on both the sphere and cylinder, because of the singlet property of the wave function, we expect the PES levels to be multiplets of the $S^{2}$ operator [65], as can be verified in Fig. 8. The most important universal information is the counting of PES levels per momentum sector, which we find to be in agreement with Ref. [62]. As we restore the $L^{2}$ degeneracy in the PES given in Ref. [62], the counting for the first three sectors is a single level with $S=1$ and $L^{z}=22$, four levels at $L_{z}=21$ (two with $S=1$ and two with $S=0$ ), and ten levels at $L_{z}=20$ (one of them with $S=2$, six with $S=1$, and three with $S=0$ ). While the finite-size splitting between these levels is nonuniversal (and differs between sphere and cylinder), we indeed obtain the identical counting per sector (Fig. 8).

\section{NASS state}

Another class of non-Abelian spin-singlet states has been proposed by Ardonne et al. [63] under the name "nonAbelian spin singlet" states (NASS). The bosonic family of such states occurs at filling factors $\nu=2 k / 3$. According to 

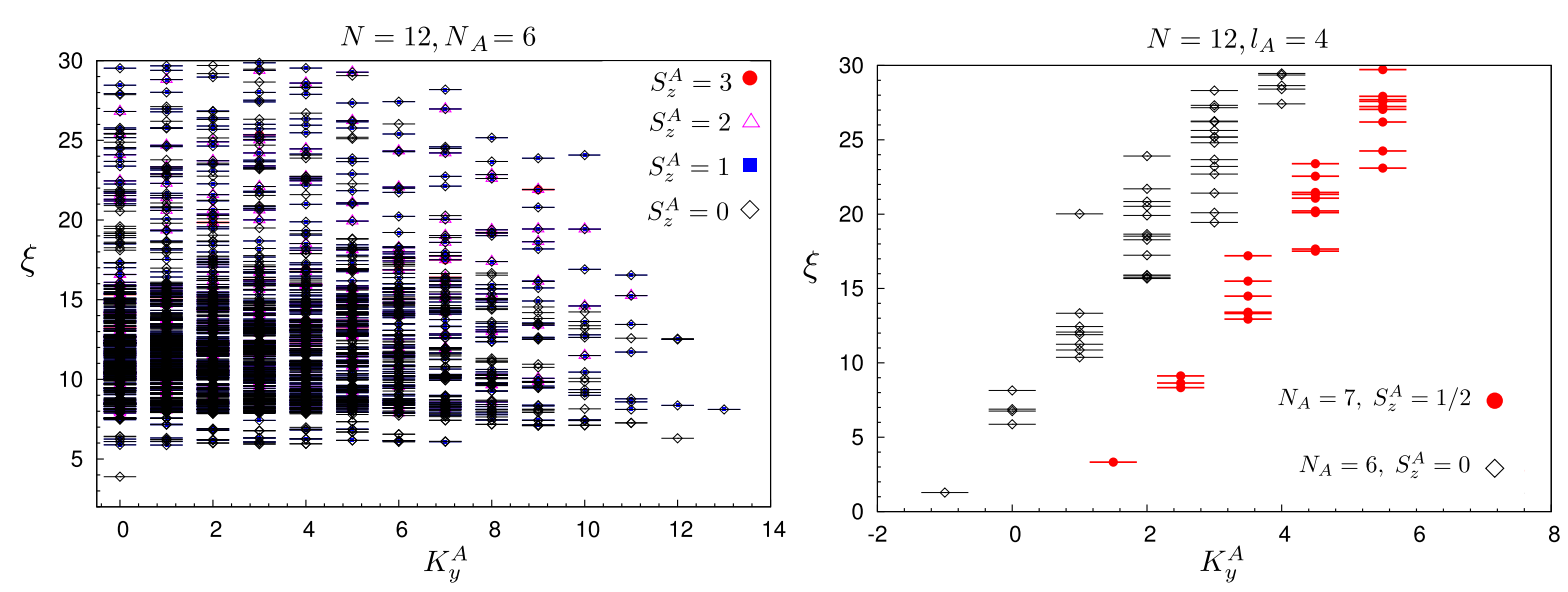

FIG. 9. PES (left panel) and OES (right panel) for the NASS $\nu=4 / 3$ state. The system contains $N=12$ bosons on a finite cylinder with aspect ratio 1 . For PES, the subsystem $A$ contains $N_{A}=6$ particles, and PES is plotted as a function of momentum $K_{y}^{A}$ of subsystem $A$. The states are labeled by different values of $S_{z}^{A}$, the total $z$ component of spin in part $A$, as indicated in the inset. For OES, the subsystem $A$ contains $l_{A}=4$ orbitals, where we show data for $N_{A}=6$ and $N_{A}=7$. The spectrum is resolved as a function of momentum $K_{y}^{A}$ of subsystem $A$.

Ref. [64], the NASS wave function ought to vanish quadratically when we bring together $k+1$ particles of the same spin, and linearly for $k+1$ particles of different spins. For $S U(2)$ spins, such wave functions only exist in the $[k+1]$ and $[k, 1]$ representations. Its parent Hamiltonian should therefore contain the PPs $U_{m=0, S=k / 2}^{k}$ and $U_{m=0, S=k / 2-1}^{k}$ since the simplest nontrivial totally or partially symmetric polynomials are of degrees two and one, respectively. Explicitly, the $b_{n}^{m=0 \dagger}$ operators are

$$
\begin{gathered}
b_{n, S=k / 2}^{0 \dagger}|0\rangle=e^{-\left(\kappa^{2} / 2\right) \sum_{i}^{k} \bar{n}_{i}^{2}|\uparrow \uparrow \ldots \uparrow\rangle,} \\
b_{n, S=k / 2-1}^{0 \dagger}|0\rangle=e^{-\left(\kappa^{2} / 2\right) \sum_{i}^{k} \bar{n}_{i}^{2}[|\uparrow \uparrow \ldots \uparrow \downarrow\rangle+|\uparrow \uparrow \ldots \downarrow \uparrow\rangle} \\
+|\uparrow \uparrow \ldots \downarrow \uparrow \uparrow\rangle+\cdots+|\downarrow \uparrow \ldots \uparrow\rangle],
\end{gathered}
$$

and similarly for the terms $S \rightarrow-S$ obtained by exchanging $|\uparrow\rangle \leftrightarrow|\downarrow\rangle$.

The simplest member $k=2$ of the NASS family has recently been studied from the "squeezing" perspective [64]. Below, we complement these results by independently generating the NASS $k=2$ state using its parent Hamiltonian in Eqs. (53) and (54). We compute its PES and OES on a finite cylinder, Fig. 9. The PES and OES are computed for $N=12$ bosons on a cylinder with aspect ratio 1. Similar to the previous cases, the universal information in the PES spectrum-the counting of levels per momentum sector-is in agreement with the independently obtained data in Ref. [64] on the sphere. In this case, the bipartition of the system is obtained by fixing the cut such that there are $l_{A}=4$ orbitals in part $A$. We compute the OES for two choices of the total number of particles in $A, N_{A}=6$ and $N_{A}=7$. We see that the counting of the OES changes as a function of $N_{A}$, as it often happens for
non-Abelian states where moving the cut probes different topological sectors of the theory, in this case associated with $\mathrm{SU}(3)_{2}$ CFT.

\section{Halperin permanent states}

Finally, we tackle more complex examples of nonAbelian states that involve a product of Halperin multicomponent states [66] and the permanent state. To be specific, we study states of the following form in the disk geometry:

$$
\Phi_{l l n}=\operatorname{Per}\left(\frac{1}{z_{i}-w_{j}}\right) \psi_{l l n}(\{z\},\{w\})
$$

where $z_{i}, w_{i}$ refer to the spin-up and spin-down particle coordinates, respectively, and $\psi_{l l n}$ is the Halperin wave function [66], an example of which was given in Eq. (41) for $l=3, n=2$. We have suppressed, as usual, the spinor and Gaussian parts of the wave function. The permanent was defined in Eq. (42).

We are particularly interested in the states $\Phi_{111}$ and $\Phi_{221}$. The former was introduced by Moore and Read [8] and subsequently analyzed in detail by Read and Rezayi [11] (see also Ref. [60]). As a prototypical example of a state that derives from a nonunitary CFT, it was shown to describe a critical point between the ferromagnet and paramagnet. Its thin-torus limit was recently studied in Ref. [39]. The latter state, $\Phi_{221}$, has been addressed by Ardonne and Regnault [64], who computed some of its properties by identifying its root configuration on the sphere and deriving its "squeezing" properties. Here, we show how to write down the parent Hamiltonian for these two states for the cylinder and torus geometry. This is of particular interest in the case of $\Phi_{221}$, where such a 

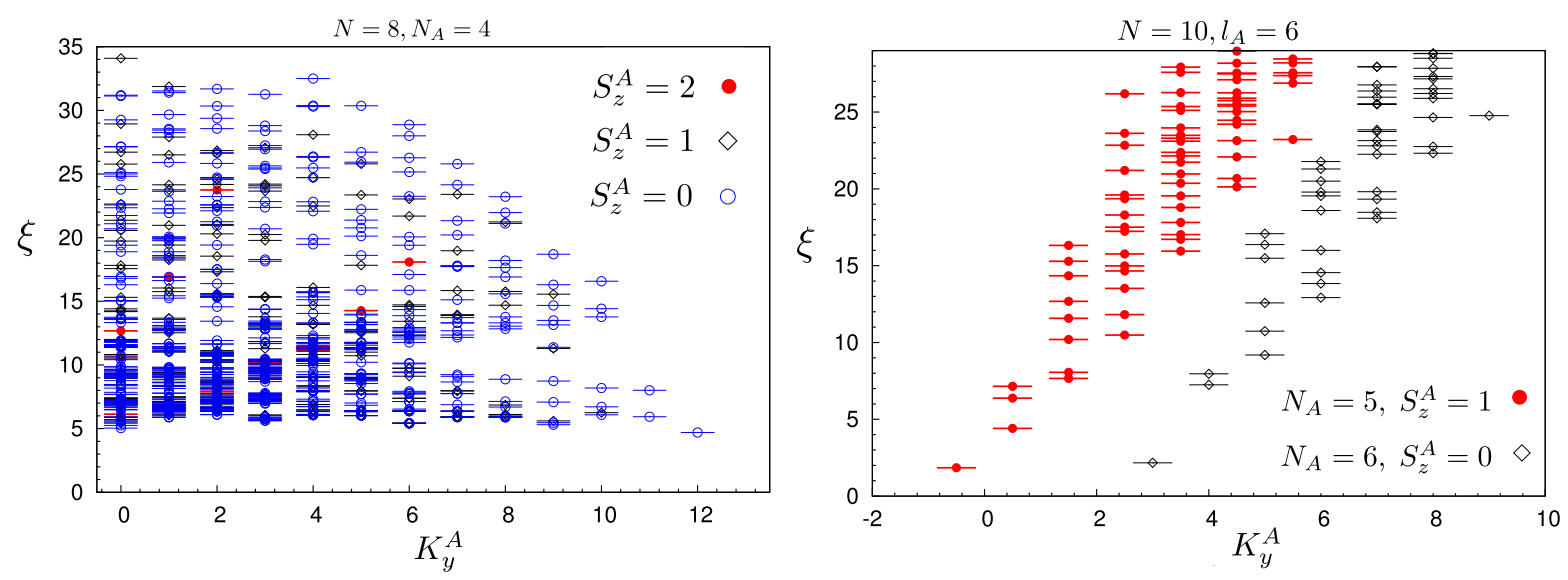

FIG. 10. PES (left panel) and OES (right panel) for the $\Psi_{221}$ state on a finite cylinder with aspect ratio equal to 1 . For PES, we consider $N=8$ bosons, and the subsystem $A$ contains $N_{A}=4$ particles. The PES is plotted as a function of momentum $K_{y}^{A}$ of subsystem $A$. Different values of $S_{z}^{A}$, the total $z$ component of spin in part $A$, are indicated in the inset. For OES, we consider $N=10$ bosons, the subsystem $A$ contains $l_{A}=6$ orbitals, and the OES is plotted as a function of momentum $K_{y}^{A}$ of subsystem $A$, for two values of the total number of particles in subsystem $A\left(N_{A}=5\right.$ and $\left.N_{A}=6\right)$.

Hamiltonian has not been reported for any geometry before.

To begin with, we want to derive many-body projection Hamiltonians whose null space contains $\Phi_{l l n}$. We first consider two-body interactions. From Eq. (55), $\Phi_{l l n}$ vanishes as the $l$ th power when $z_{i} \rightarrow z_{j}$ (or $w_{i} \rightarrow w_{j}$ ). Hence, we need two-body terms $U_{m, S=1}^{2}$, where $m<l$ and $|S|=1$, i.e., in the $|\uparrow \uparrow\rangle$ or $|\downarrow \downarrow\rangle$ channel. In the $S=0$ (or $|\uparrow \downarrow\rangle$ ) channel, however, one term in the permanent state will cause $\Phi_{l l n}$ not to vanish for $z_{i} \rightarrow w_{k}$. Hence, we do not need any $U_{m, S=0}^{2}$.

For three-body interactions, we find that the Halperinfunction parts contribute a degree of $l+2 n$ for $S=\frac{1}{2}$ (or the $|\uparrow \uparrow \downarrow\rangle,|\uparrow \downarrow \downarrow\rangle$, etc.) channels. Also, the permanent state will remove one degree. Hence, we need $U_{m, 1 / 2}^{3}$ for $m<l+2 n-1$. An analogous analysis for $|\uparrow \uparrow \uparrow\rangle$ or $|\downarrow \downarrow \downarrow\rangle$ reveals that we also need $U_{m, 3 / 2}^{3}$ for $m<3 l$. The matter simplifies a bit since not all of the requisite $U_{m, S}^{2,3}$ mentioned above actually exist. In Sec. IV and also Table 1 of Ref. [43], the PPs $U_{m, S}^{2}$ are nonzero for even $m$ when $l$ is even and $S=1$, or $l$ odd and $S=0$ (and vice versa for total antisymmetry in spin-orbit space). For $N=3, U_{m, 1 / 2}^{3}$ exists for $m \geq 1$, but $U_{m, 3 / 2}^{3}$ does not exist for $m=0,1,2$ in the case of fermions (odd $l$ ). In summary, the above considerations for $n=1$ and $l=1,2$ imply that only $U_{1,1 / 2}^{3}$ is required to produce $\Phi_{111}$, consistent with Ref. [11]. On the other hand, $U_{0,1}^{2}, U_{1,1 / 2}^{3}, U_{2,1 / 2}^{3}$, and $U_{m, 3 / 2}^{3}$ for $m=$ $0,2,3,4,5$ are all required to produce $\Phi_{221}$. Therefore, we see that formulating the parent Hamiltonian for $\Phi_{221}$ is indeed rather involved and crucially benefits from the systematic approach we follow here.

For completeness, we quote the explicit expressions for these two Hamiltonians in the form
$U_{m, S}^{N}=\sum_{n} b_{n}^{m, S \dagger} b_{n}^{m, S}+(|\uparrow\rangle \leftrightarrow|\downarrow\rangle)$. For the $\Phi_{111}$ state, we have

$$
H_{111}=U_{1,1 / 2}^{3} \propto \sum_{n} b_{n}^{1,1 / 2 \dagger} b_{n}^{1,1 / 2}
$$

with the corresponding operator and polynomial amplitude

$$
\begin{aligned}
b_{n}^{1,1 / 2}=\kappa^{3 / 2} & \sum_{\sum n_{i}=n} p_{1,1 / 2}(\kappa ;\{n\}) e^{-\left(\kappa^{2} / 3\right) W^{2}} c_{n_{1}} c_{n_{2}} c_{n_{3}}, \\
p_{1,1 / 2}= & \left(\bar{n}_{1}-\bar{n}_{2}\right)|\uparrow \uparrow \downarrow\rangle+\left(\bar{n}_{2}-\bar{n}_{3}\right)|\downarrow \uparrow \uparrow\rangle \\
& +\left(\bar{n}_{3}-\bar{n}_{1}\right)|\uparrow \downarrow \uparrow\rangle,
\end{aligned}
$$

where as before $\bar{n}_{i}=n_{i}-\frac{1}{3} \sum_{j} n_{j}$. For $\Phi_{221}$, we have

$$
\begin{aligned}
H_{221}= & U_{0,1}^{2}+\sum_{m=1,2} \gamma_{m} U_{m, 1 / 2}^{3} \\
& +\sum_{m=0,2,3,4,5} \eta_{m} U_{m, 3 / 2}^{3},
\end{aligned}
$$

where again $\gamma_{m}$ and $\eta_{m}$ can be chosen to be arbitrary positive constants.

We have numerically diagonalized the Hamiltonian (59) and verified that it gives a unique zero-energy ground state on finite cylinders when $N_{\phi}=3 N / 2-3$. By stretching the cylinder, we confirmed that the ground state reduces to the pattern $(\uparrow, \downarrow) 00(\uparrow, \downarrow) 00 \ldots$, which coincides with the root configuration given in Ref. [64]. In Fig. 10 (left panel), we compute the PES for $N=8$ bosons, which can be directly compared with Fig. 7 in Ref. [64]. As expected, we find an agreement in the counting of the PES levels between our results on the cylinder and the sphere data in Ref. [64]. Finally, we also provide a plot of the OES in Fig. 10 (right 
panel). As shown by Li and Haldane [25], a non-Abelian state like the Moore-Read state will have different counting depending on the position of the orbital entanglement cut, which effectively probes different topological sectors of the theory. This is also the case in our example, as we see the counting changes depending on the number of particles in subsystem $A$ while the location of the cut remains fixed. Furthermore, the spectrum is fully chiral, which suggests that this state may be described by a simple CFT as conjectured in Ref. [64]. The CFT can, in principle, be identified using the counting of the OES in Fig. 10. For this purpose, larger systems may be required in order to verify that the counting we see in Fig. 10 is saturated and indeed corresponds to the thermodynamic limit, which we defer to future work.

\section{CONCLUSION}

We have provided a general method for constructing quantum Hall parent Hamiltonians with the desired type of clustering properties on cylinder and torus geometries. The approach developed here completes the program initiated in Ref. [30], which is suited for the disk and sphere geometry that have a conserved $z$ component of angular momentum. We have performed extensive analytic and numerical checks of the proposed model Hamiltonians and generally find complete agreement with previous results if available. We have also demonstrated that it is possible to construct the parent Hamiltonians for some rather complex spinful non-Abelian states, which previously have not been provided in the literature.

The method presented here opens up several future directions. One can use the Hamiltonians derived here to systematically scan for filling factors where the Hamiltonians have zero-energy ground states, in the spirit of Ref. [67]. Performing this kind of search is much more natural in the torus geometry, which does not suffer from the known shift bias [68]. Furthermore, our approach straightforwardly generalizes to four-body interactions whose possible incompressible ground states have not been systematically investigated before. Another interesting extension to pursue is the study of multicomponent states beyond the familiar SU(2) spin, such as the SU(4) valley or spin symmetric non-Abelian states. They may have relevance in the realization of FQHE in graphene, where the open accessibility of the electron gas may allow for further tunability of the interaction profile $[69,70]$.

\section{ACKNOWLEDGMENTS}

C. H. L. is supported by a NSS grant from the Agency of Science, Technology and Research of Singapore. Z. P. acknowledges support from U.S. Department of Energy Grant No. DE-SC0002140. Research at Perimeter Institute is supported by the Government of Canada through Industry Canada and by the Province of Ontario through the Ministry of Economic Development \& Innovation. R. T. is supported by the European Research Council through Grant No. ERC-StG-TOPOLECTRICS-336012.

\section{APPENDIX A: BARYCENTRIC COORDINATES FOR MANY-BODY PSEUDOPOTENTIALS}

We describe how to find a manifestly $S_{N}$-symmetric embedding of the tuple $\left(\bar{n}_{1}, \ldots, \bar{n}_{N}\right)$ onto the $(N-1)$ dimensional simplex in $\mathbb{R}^{N-1}$. This can be achieved in barycentric coordinates, which is also useful for diverse applications involving permutation symmetry with a linear constraint [71]. The most straightforward construction is to write

$$
\vec{x}=\kappa \sum_{k=1}^{N} \vec{n}_{k} \vec{\beta}_{k},
$$

where $\vec{x} \in \mathbb{R}^{N-1}$ and $\left\{\vec{\beta}_{k}\right\}, k=1, \ldots, N$ form a linearly dependent set of basis vectors, also in $\mathbb{R}^{N-1}$, normalized so that

$$
\vec{\beta}_{j} \cdot \vec{\beta}_{k}=\frac{N}{N-1} \delta_{j k}-\frac{1}{N-1} .
$$

Geometrically, the $\beta_{k}$ 's define the vertices of a simplex and are at an angle of $\cos ^{-1}[-1 /(N-1)]$ from one another. The vertex $k$ corresponds to the least isotropic configuration with $\bar{n}_{k} \propto(N-1) /(N)$ and $\bar{n}_{j} \propto-(1 / N), j \neq k$. A basis consistent with the above requirements is

$$
\begin{aligned}
\vec{\beta}_{1} & =(1,0, \ldots, 0), \\
\vec{\beta}_{2} & =\left(C_{1}, S_{1}, \ldots, 0\right), \\
\vec{\beta}_{3} & =\left(C_{1}, S_{1} C_{2}, S_{1} S_{2}, \ldots, 0\right), \\
\vec{\beta}_{4} & =\left(C_{1}, S_{1} C_{2}, S_{1} S_{2} C_{3}, S_{1} S_{2} S_{3}, \ldots, 0\right), \\
& \vdots \\
\vec{\beta}_{N-1} & =\left(C_{1}, S_{1} C_{2}, S_{1} S_{2} C_{3}, \ldots, S_{1} \ldots S_{N-2}\right), \\
\vec{\beta}_{N} & =\left(C_{1}, S_{1} C_{2}, S_{1} S_{2} C_{3}, \ldots,-S_{1} \ldots S_{N-2}\right),
\end{aligned}
$$

with $S_{k}^{2}+C_{k}^{2}=1,1 \leq k \leq N-2$. Upon enforcing the scalar product constraint in Eq. (A2), we require that $\left.S_{k+1}=1-[1+1 / N) /\left(\prod_{j=1}^{k} S_{k}\right)^{2}\right]$, which also implies that $C_{k}^{2}+S_{k}^{2} C_{k+1}=C_{k}$. A simple solution fortunately exists:

$$
C_{k}=-\frac{1}{N-k},
$$


which implies that the $k$ th projected component of the relative angles between the position vectors of the vertices approaches $\pi$ as $k$ and $N$ increase. We proceed by substituting Eq. (A4) into the explicit form of vertex positions $\vec{\beta}_{k}$, and expressing the latter in terms of the spherical coordinates. From Eq. (A1), we can easily check that

$$
\bar{n}_{k}=\frac{N-1}{N \kappa} \vec{x} \cdot \vec{\beta}_{k}
$$

and that

$W^{2}=|\vec{x}|^{2}=\frac{N \kappa^{2}}{N-1} \sum_{k}^{N}\left(n_{k}-\frac{n}{N}\right)^{2}=\frac{N \kappa^{2}}{N-1} \sum_{k}^{N} \bar{n}_{k}^{2}$.

For the purpose of orthogonalizing the PPs over the inner product measure in Eq. (14), we also need to express $\vec{x}$ explicitly in terms of angles in origin-centered spherical coordinates:

$$
\begin{aligned}
x_{1} & =W \cos \varphi_{1}, \\
x_{2} & =W \sin \varphi_{1} \cos \varphi_{2}, \\
\vdots & \\
x_{N-2} & =W \cos \varphi_{k} \prod_{k=1}^{N-3} \sin \varphi_{k}, \\
x_{N-1} & =W \prod_{k=1}^{N-2} \sin \varphi_{k} .
\end{aligned}
$$

Substituting the explicit expressions from Eqs. (A3) and (A7) into Eq. (A5), we obtain

$$
\begin{gathered}
\bar{n}_{1}=\frac{N-1}{N \kappa} W \cos \varphi_{1}, \\
\bar{n}_{2}=\frac{W}{N \kappa}\left(-\cos \varphi_{1}+\sqrt{N(N-2)} \sin \varphi_{1} \cos \varphi_{2}\right), \\
\bar{n}_{k}=\frac{W}{N \kappa}\left[-\cos \varphi_{1}-\sqrt{\frac{N}{N-2}} \sin \varphi_{1} \cos \varphi_{2}\right. \\
-\sum_{j=2}^{k-2} \sqrt{\frac{N !}{(N-j-2) !} \frac{\left(\prod_{i=1}^{j} \sin \varphi_{i}\right) \cos \varphi_{j+1}}{(N-j)(N-j-1)}} \\
\left.+\sqrt{\frac{N !}{(N-k-1) !}} \frac{\left(\prod_{i=1}^{k-1} \sin \varphi_{i}\right) \cos \varphi_{k}}{N-k+1}\right],
\end{gathered}
$$

$$
\begin{aligned}
\bar{n}_{N-1}= & \frac{W}{N \kappa}\left[-\cos \varphi_{1}-\sqrt{\frac{N}{N-2}} \sin \varphi_{1} \cos \varphi_{2}\right. \\
& -\sum_{j=2}^{N-3} \sqrt{\frac{N !}{(N-j-2) !}} \frac{\left(\prod_{i=1}^{j} \sin \varphi_{i}\right) \cos \varphi_{j+1}}{(N-j)(N-j-1)} \\
& \left.+\frac{\sqrt{N !}}{2}\left(\prod_{i=1}^{N-2} \sin \varphi_{i}\right)\right], \\
\bar{n}_{N}= & \frac{W}{N \kappa}\left[-\cos \varphi_{1}-\sqrt{\frac{N}{N-2}} \sin \varphi_{1} \cos \varphi_{2}\right. \\
& -\sum_{j=2}^{N-3} \sqrt{\frac{N !}{(N-j-2) !} \frac{\left(\prod_{i=1}^{j} \sin \varphi_{i}\right) \cos \varphi_{j+1}}{(N-j)(N-j-1)}} \\
& \left.-\frac{\sqrt{N !}}{2}\left(\prod_{i=1}^{N-2} \sin \varphi_{i}\right)\right]
\end{aligned}
$$

These are the explicit expressions for transcribing the $\bar{n}_{j}$, $1 \leq j \leq N$ indices directly into spherical coordinates. In Eq. (A10), $k$ ranges from 3 to $N-2$.

\section{APPENDIX B: SECOND-QUANTIZED PSEUDOPOTENTIALS VS REAL-SPACE PROJECTION HAMILTONIANS}

We provide a brief comparison between the secondquantized PPs that have been derived in this paper and realspace projection Hamiltonians elsewhere in the literature, e.g., Trugman-Kivelson-type Hamiltonians on the infinite plane. We show that our geometric PP construction avoids certain ambiguities that plague the latter approaches.

Neglecting internal DOFs for simplicity, a generic realspace PP living in total relative angular-momentum sectors up to $m$ takes the form

$H\left(\vec{r}_{1}, \ldots, \vec{r}_{N}\right)=\left(\prod_{j=1}^{N} \nabla_{j}^{2 d_{j}}\right) \delta^{2}\left(\vec{r}_{1}-\vec{r}_{2}\right) \ldots \delta^{2}\left(\vec{r}_{N-1}-\vec{r}_{N}\right)$

such that $\sum_{j} d_{j}=m$. The various $d_{j}$ 's refer to how the derivatives are distributed among the particles. In this form, there is no simple one-to-one correspondence between the $d_{j}$ values and the relative weight of $H$ in the various $m$ sectors. To find the relative weights, one has to project $H$ onto the sector spanned by a state with angular momentum $m$. It is most convenient to adopt the symmetric gauge, where such a state takes the form $\left|\Psi_{m}\right\rangle \propto p\left(\tilde{z}_{1}, \ldots, \tilde{z}_{N}\right) e^{-\frac{1}{4} \sum_{j}\left|z_{j}\right|^{2}}\left|z_{1}, \ldots, z_{N}\right\rangle$, where $p$ is a symmetric or antisymmetric polynomial and $z_{j}=x_{j}+i y_{j}$ refers to the particle position ( $\tilde{z}$ defined below): 


$$
\left\langle\Psi_{m}|H| \Psi_{m}\right\rangle=\left(\prod_{j=1}^{N} \int d^{2} \vec{r}_{j}\right) \Psi_{m}^{*}\left(\vec{r}_{1}, \ldots, \vec{r}_{N}\right) H\left(\vec{r}_{1}, \ldots, \vec{r}_{N}\right) \Psi_{m}\left(\vec{r}_{1}, \ldots, \vec{r}_{N}\right)
$$

Upon substituting $\Psi_{m}$ and integrating by parts, we can express $\left\langle\Psi_{m}|H| \Psi_{m}\right\rangle$ as

$$
\left\langle\Psi_{m}|H| \Psi_{m}\right\rangle=\left(\prod_{j=1}^{N} \int d^{2} \vec{r}_{j}\right) \delta^{2}\left(\vec{r}_{1}-\vec{r}_{2}\right) \ldots \delta^{2}\left(\vec{r}_{N-1}-\vec{r}_{N}\right) \times\left(\prod_{j=1}^{N} \nabla_{j}^{2 d_{j}}\right)\left[\Psi_{m}^{*}\left(\vec{r}_{1}, \ldots, \vec{r}_{N}\right) \Psi_{m}\left(\vec{r}_{1}, \ldots, \vec{r}_{N}\right)\right],
$$

which can be evaluated as

$$
\left\langle\Psi_{m}|H| \Psi_{m}\right\rangle=4^{m}\left(\prod_{j=1}^{N} \int d z_{j} d z_{j}^{*}\right) \delta^{2}\left(z_{1}-z_{2}\right) \ldots \delta^{2}\left(z_{N-1}-z_{N}\right)\left(\prod_{j=1}^{N} \partial_{z_{j}^{*}}^{2 d_{j}} \Psi_{m}^{*}\left(z_{1}^{*}, \ldots, z_{N}^{*}\right)\right)\left(\prod_{j=1}^{N} \partial_{z_{j}}^{2 d_{j}} \Psi_{m}\left(z, \ldots, z_{N}\right)\right),
$$

where $\nabla^{2}=4 \partial_{z} \partial_{\bar{z}}$ has been used. The delta functions, which enforce that all $z_{j}$ be equal, also enforce $\tilde{z}_{j}=z_{j}-(1 / N) \sum_{j} z_{j}=0, \forall j$. Thus, we only get a nonzero contribution from constant integrands. Specific examples were worked out in the appendixes of Ref. [42]; in general, an operator $H$ has an overlap with various $m$ sectors, and a PP that lies purely in one $m$ sector must be a complicated linear combination of the Trugman-Kivelson terms with various sets of $d_{j}$ 's. Some progress was made in Ref. [44], where operators containing $L_{m}\left(N \kappa^{2} / 2(N-1) \nabla_{1}^{2}\right)$ were shown to be contained purely in one $m$ sector. That, however, does not always work for fermions, as will be explained below.

The mathematical complications above are further aggravated when the space of PPs in the sector $m$ is degenerate. In particular, the matrix $\left\langle\Psi_{m a}|H| \Psi_{m b}\right\rangle$ is not of full rank, and some combinations of bona-fide PPs of degree $m$ will still not give a positive energy penalty to states in the $m$ sector [42]. In particular, it is possible for certain TrugmanKivelson Hamiltonians with $2 m$ derivatives to evaluate to zero upon taking into account the fermionic antisymmetry. Consider, for instance, the $N=3$ fermionic state $\left|\Psi_{3}\right\rangle \propto\left(\tilde{z}_{1}-\tilde{z}_{2}\right)\left(\tilde{z}_{2}-\tilde{z}_{3}\right)\left(\tilde{z}_{3}-\tilde{z}_{1}\right)\left|z_{1}, z_{2}, z_{3}\right\rangle$. The following operators have a zero projection on it:

$$
\left\langle\Psi_{3}\left|\nabla_{j}^{6} \delta^{2}\left(r_{1}-r_{2}\right) \delta^{2}\left(r_{2}-r_{3}\right)\right| \Psi_{3}\right\rangle=0
$$

and

$$
\left\langle\Psi_{3}\left|\nabla_{1}^{2} \nabla_{2}^{2} \nabla_{3}^{2} \delta^{2}\left(r_{1}-r_{2}\right) \delta^{2}\left(r_{2}-r_{3}\right)\right| \Psi_{3}\right\rangle=0 .
$$

Indeed, the only nonvanishing Trugman-Kivelsontype fermionic PP in the $U_{m=3}^{N=3}$ sector is $H=$ $\nabla_{i}^{4} \nabla_{j}^{2} \delta^{2}\left(r_{1}-r_{2}\right) \delta^{2}\left(r_{2}-r_{3}\right)$. As $\left|\Psi_{3}\right\rangle$ is of degree $\leq 2$ in each variable, $H$ must contain a derivative of degrees $2 d_{j} \leq 4$ in each variable for a nonzero projection. Since only constant terms in the integrand of Eq. (B4) contribute, we also need derivatives in all three particle coordinates.
By contrast, our PP construction appeals to the orthogonality structure of the PPs from the outset, and it avoids all of the above problems that generically arise when one attempts to use the Trugman-Kivelson approach in more complicated many-body cases.

\section{APPENDIX C: PSEUDOPOTENTIAL PARENT HAMILTONIAN FROM A GIVEN WAVE FUNCTION}

We briefly summarize the heuristic procedure for how to determine the PPs whose null spaces contain a given $\mathrm{QH}$ state. These are the PPs that should be included in its parent Hamiltonian since they penalize denser or "simpler" states that will otherwise be realized at the same filling fraction. The method consists of two steps: (i) identify the thin-torus root pattern from the first-quantized wave function (Sec. C 1), and (ii) use the root pattern and power counting to obtain the PPs (Sec. C 2).

\section{Wave function to root pattern}

Suppose we are given a $\mathrm{QH}$ wave function with the polynomial part $\psi\left(z_{1}, z_{2}, \ldots\right)$, where the $z_{i}$ 's denote the positions of particles. In the infinite plane, $z_{j}$ is the usual complex coordinate $z_{j}=x_{j}+i y_{j}$. Since we will work on a cylinder geometry, however, we need to perform a stereographic mapping: $z_{j} \rightarrow e^{\kappa z_{j}}$, where $\kappa=2 \pi / L_{y}$ is shown in

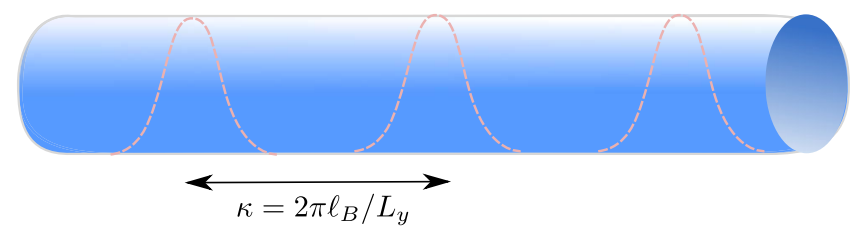

FIG. 11. In the limit of a thin cylinder $(\kappa \rightarrow \infty)$, the singleparticle orbitals become well separated from one another. As a consequence, the dominant configurations ("root patterns") in the expansion of a many-body state $\Psi$ are those Fock states where particles minimize their classical electrostatic energy. 
Fig. 11. For simplicity, assume there are no internal degrees of freedom.

To each wave function defined on a cylinder, we can assign one or several thin-cylinder root patterns. Those are the Fock states with nonzero weight as the circumference of the cylinder is taken to zero $(\kappa \rightarrow \infty)$. Typically, any given $\mathrm{FQH}$ state $|\psi\rangle$ in the thermodynamic limit decomposes onto many Fock states, as $|\psi\rangle$ represents a strongly correlated state. However, when the cylinder is stretched, the weights of most of the Fock states in the decomposition vanish. The only Fock states surviving in the limit $\kappa \rightarrow \infty$ are called "root patterns" and are useful for finding the parent Hamiltonian (Laplacian) for $|\psi\rangle$ as its kernel.

In order to find the root pattern, we must examine the dominant terms in the decomposition of $\psi$ as $\kappa \rightarrow \infty$. Usually, this amounts to solving a classical electrostatic problem. Here, we consider the case of an open cylinder, where the root pattern will typically be unique. To get the remaining root patterns that arise because of topological degeneracy or non-Abelian statistics, one must consider the system on a torus. Because of the complicated form of torus wave functions, it is more convenient to work on a cylinder but perform a flux insertion that allows one to access other topological sectors. Details of this are given, e.g., in Ref. [56].

Fixing the total number of particles, we study the dominating term in $\psi$. It is $z_{1}^{m_{1}} z_{2}^{m_{2}} z_{3}^{m_{3}} \ldots$ such that no other term (denoted by ') has $m_{1}^{\prime}>m_{1}$, or $m_{2}^{\prime}>m_{2}$ if $m_{1}^{\prime}=m_{1}$, or $m_{3}^{\prime}>m_{3}$ if $m_{1}^{\prime}=m_{1}$ and $m_{2}^{\prime}=m_{2}$, etc. The root pattern can then be written down as the partition defined by $m_{1}, m_{2}, \ldots$ :

$$
\left(m_{1}, m_{2}, m_{3}, \ldots\right) \rightarrow 10 \ldots 010 \ldots 01 \ldots
$$

where the 1's are at position $m_{1}, m_{2}, \ldots$. If two $m_{i}$ 's coincide, as can occur for bosons or multicomponent fermions, we label that position by " 2 " and so forth. For instance, consider the $1 / \mathrm{m}$ Laughlin state with two particles. We have $\psi\left(z_{1}, z_{2}\right)=\left(z_{1}-z_{2}\right)^{m}$ which, when expanded out, contains monomials

$$
\left\{z_{1}^{m}, z_{1}^{m-1} z_{2}, z_{1}^{m-2} z_{2}^{2}, \ldots\right\}
$$

Evidently, the term $z_{1}^{m} z_{2}^{0}$ is in the root pattern. Had we considered a droplet of $N$ particles, this term would have become $z_{1}^{m(N-1)} z_{2}^{m(N-2)} \ldots z_{N-1}^{m} z_{N}^{0}$, with total degree $m N(n-1) / 2$ and the root

$$
10^{m-1} 10^{m-1} 1 \ldots
$$

where $0^{m-1}$ represents a string of $m-1$ consecutive zeros. For this simplest case, a droplet of $N=2$ particles is sufficient to determine the root configuration. Because we deal with translationally invariant states, a root configuration for larger $N$ is obtained by repeating the minimal pattern.

Let us now consider a more complicated example such as the generalized Pfaffian state introduced in Eq. (46) in Sec. V. By using the identity $[P f(A)]^{2}=\operatorname{Det}(A)$, one can show by induction, starting from $n=4$, the most compact particle droplet, that the dominating term is $z_{1}^{3 q-1} z_{2}^{2 q} z_{3}^{q-1}$. This defines the root configuration in Eq. (47), $10^{q-2} 10^{q} 10^{q-2} 10^{q} \ldots$ It is also easy to read off the filling fraction from the above: We have two particles per period, and each period has length $2 q$. Hence, the filling fraction is $(2 / 2 q)=1 / q$.

\section{Root pattern to parent Hamiltonian}

Given a root pattern, we can easily infer what relative angular-momentum states should be penalized to construct a projector onto the state. For instance, if the closest pair of particles is ...10 $10^{l} 1 \ldots$, no two particles have relative angular momentum less than $l+1$. Hence, the state should lie in the null space of any two-body PPs with $U_{m}^{2}, m \leq l$. Consequently, the parent Hamiltonian should be a linear combination of these PPs so that it penalizes all states denser than the given one. This is discussed at length in Ref. [30].

To figure out the required many-body PPs, we apply the above procedure analogously to clusters of particles with minimal relative angular momentum. Note that some manybody PPs need not be included if the interaction is already precluded by particle (anti)symmetry: Consider the $1 / q$ Pfaffian state described above, with a root given by Eq. (47). Obviously, no two particles have relative angular momentum less than $q-1$. Naively, we then need to include all $U_{m}^{2}$ with $m \leq q-2$. The $1 / q$ Pfaffian state, however, is fermionic (bosonic) when $q$ is even (odd), and $U_{q-2}^{2}$ exactly vanishes in both cases. Hence, the parent Hamiltonian only contains $U_{m<q-2}^{2}$.

Let us finally analyze three-body interactions. From the root pattern, the minimal total relative angular momentum is $l=(q-1)+2 q=3 q-1$. However, configurations with $l=3 q-2=2(q-1)+q$ automatically vanish since they require pairs of particles with relative angular momenta $q$ and $q-1$ simultaneously, which are forbidden by either fermionic or bosonic statistics. With lower threebody relative angular momentum already excluded by the two-body PPs, the only three-body PP we need to include in the parent Hamiltonian is $U_{3 q-3}^{3}$. As the root pattern repeats from the third particle, there is no need to consider interactions with four or more bodies.

[1] D. C. Tsui, H. L. Stormer, and A. C. Gossard, TwoDimensional Magnetotransport in the Extreme Quantum Limit, Phys. Rev. Lett. 48, 1559 (1982). 
[2] R. B. Laughlin, Anomalous Quantum Hall Effect: An Incompressible Quantum Fluid with Fractionally Charged Excitations, Phys. Rev. Lett. 50, 1395 (1983).

[3] F. D. M. Haldane, Fractional Quantization of the Hall Effect-A Hierarchy of Incompressible Quantum Fluid States, Phys. Rev. Lett. 51, 605 (1983).

[4] F. D. M. Haldane, The Quantum Hall Effect, edited by R. E. Prange and S. M. Girvin (Springer, New York, 1990).

[5] G. Fano, F. Ortolani, and E. Colombo, ConfigurationInteraction Calculations on the Fractional Quantum Hall Effect, Phys. Rev. B 34, 2670 (1986).

[6] C. Nayak, S. H. Simon, A. Stern, M. Freedman, and S. Das Sarma, Non-Abelian Anyons and Topological Quantum Computation, Rev. Mod. Phys. 80, 1083 (2008).

[7] J. K. Pachos, Introduction to Topological Quantum Computation (Cambridge University Press, Cambridge, England, 2012).

[8] G. Moore and N. Read, Nonabelions in the Fractional Quantum Hall Effect, Nucl. Phys. B360, 362 (1991).

[9] M. Greiter, X.-G. Wen, and F. Wilczek, Paired Hall State at Half Filling, Phys. Rev. Lett. 66, 3205 (1991).

[10] M. Greiter, X.-G. Wen, and F. Wilczek, Paired Hall States, Nucl. Phys. B374, 567 (1992).

[11] N. Read and E. Rezayi, Quasiholes and Fermionic Zero Modes of Paired Fractional Quantum Hall States: The Mechanism for Non-Abelian Statistics, Phys. Rev. B 54, 16864 (1996).

[12] N. Read, Paired Fractional Quantum Hall State and the $\nu=5 / 2$ Puzzle, Physica (Amsterdam) 298B, 121 (2001).

[13] N. Read and E. Rezayi, Beyond Paired Quantum Hall States: Parafermions and Incompressible States in the First Excited Landau Level, Phys. Rev. B 59, 8084 (1999).

[14] M. Greiter and R. Thomale, Non-Abelian Statistics in a Quantum Antiferromagnet, Phys. Rev. Lett. 102, 207203 (2009).

[15] T. S. Jackson, N. Read, and S. H. Simon, Entanglement Subspaces, Trial Wave Functions, and Special Hamiltonians in the Fractional Quantum Hall Effect, Phys. Rev. B 88, 075313 (2013).

[16] G. Ortiz, Z. Nussinov, J. Dukelsky, and A. Seidel, Repulsive Interactions in Quantum Hall Systems as a Pairing Problem, Phys. Rev. B 88, 165303 (2013).

[17] L. Chen and A. Seidel, Algebraic Approach to the Study of Zero Modes of Haldane Pseudopotentials, Phys. Rev. B 91, 085103 (2015).

[18] T. Mazaheri, G. Ortiz, Z. Nussinov, and A. Seidel, Zero Modes, Bosonization, and Topological Quantum Order: The Laughlin State in Second Quantization, Phys. Rev. B 91, 085115 (2015).

[19] T. H. Hansson, M. Hermanns, and S. Viefers, Quantum Hall Quasielectron Operators in Conformal Field Theory, Phys. Rev. B 80, 165330 (2009).

[20] M. Greiter, V. Schnells, and R. Thomale, Laughlin States and Their Quasi-Particle Excitations on the Torus, arXiv:1405.0742.

[21] S. M. Girvin, A. H. MacDonald, and P. M. Platzman, Collective-Excitation Gap in the Fractional Quantum Hall Effect, Phys. Rev. Lett. 54, 581 (1985).

[22] S. M. Girvin, A.H. MacDonald, and P. M. Platzman, Magneto-Roton Theory of Collective Excitations in the Fractional Quantum Hall Effect, Phys. Rev. B 33, 2481 (1986).
[23] C. Repellin, T. Neupert, Z. Papic, and N. Regnault, SingleMode Approximation for Fractional Chern Insulators and the Fractional Quantum Hall Effect on the Torus, Phys. Rev. B 90, 045114 (2014).

[24] C. Repellin, T. Neupert, B. Andrei Bernevig, and N. Regnault, Projective Construction of the $\mathbb{Z}_{k}$ Read-Rezayi Fractional Quantum Hall States and Their Excitations on the Torus Geometry, arXiv:1504.00367.

[25] H. Li and F. D. M. Haldane, Entanglement Spectrum as a Generalization of Entanglement Entropy: Identification of Topological Order in Non-Abelian Fractional Quantum Hall Effect States, Phys. Rev. Lett. 101, 010504 (2008).

[26] R. Thomale, A. Sterdyniak, N. Regnault, and B. Andrei Bernevig, Entanglement Gap and a New Principle of Adiabatic Continuity, Phys. Rev. Lett. 104, 180502 (2010).

[27] A. Sterdyniak, N. Regnault, and B. A. Bernevig, Extracting Excitations from Model State Entanglement, Phys. Rev. Lett. 106, 100405 (2011).

[28] F. D. M. Haldane and E. H. Rezayi, Spin-Singlet Wave Function for the Half-Integral Quantum Hall Effect, Phys. Rev. Lett. 60, 956 (1988).

[29] M. Greiter, Root Configurations and Many Body Interactions for Fractionally Quantized Hall States, Bull. Am. Phys. Soc. 38, 137 (1993).

[30] S. H. Simon, E. H. Rezayi, and N. R. Cooper, Generalized Quantum Hall Projection Hamiltonians, Phys. Rev. B 75, 075318 (2007).

[31] X. G. Wen and Q. Niu, Ground-State Degeneracy of the Fractional Quantum Hall States in the Presence of a Random Potential and on High-Genus Riemann Surfaces, Phys. Rev. B 41, 9377 (1990).

[32] X.-G. Wen, Topological Orders in Rigid States, Int. J. Mod. Phys. B 04, 239 (1990).

[33] Y. Zhang, T. Grover, A. Turner, M. Oshikawa, and A. Vishwanath, Quasiparticle Statistics and Braiding from Ground-State Entanglement, Phys. Rev. B 85, 235151 (2012).

[34] D.-H. Lee and J. M. Leinaas, Mott Insulators without Symmetry Breaking, Phys. Rev. Lett. 92, 096401 (2004).

[35] A. Seidel, H. Fu, D.-H. Lee, J. M. Leinaas, and J. Moore, Incompressible Quantum Liquids and New Conservation Laws, Phys. Rev. Lett. 95, 266405 (2005).

[36] S. Jansen, Fermionic and Bosonic Laughlin State on Thick Cylinders, J. Math. Phys. (N.Y.) 53, 123306 (2012).

[37] Z.-Y. Wang and M. Nakamura, One-Dimensional Lattice Model with an Exact Matrix-Product Ground State Describing the Laughlin Wave Function, Phys. Rev. B 87, 245119 (2013).

[38] P. Soulé and T. Jolicoeur, Exact Wave Functions for Excitations of the $\nu=\frac{1}{3}$ Fractional Quantum Hall State from a Model Hamiltonian, Phys. Rev. B 85, 155116 (2012).

[39] Z. Papic, Solvable Models for Unitary and Nonunitary Topological Phases, Phys. Rev. B 90, 075304 (2014).

[40] A. Seidel and K. Yang, Gapless Excitations in the HaldaneRezayi State: The Thin-Torus Limit, Phys. Rev. B 84, 085122 (2011).

[41] A. Weerasinghe and A. Seidel, Thin Torus Perturbative Analysis of Elementary Excitations in the Gaffnian and 
Haldane-Rezayi Quantum Hall States, Phys. Rev. B 90, 125146 (2014).

[42] S. H. Simon, E. H. Rezayi, and N. R. Cooper, Pseudopotentials for Multiparticle Interactions in the Quantum Hall Regime, Phys. Rev. B 75, 195306 (2007).

[43] S. C. Davenport and S. H. Simon, Multiparticle Pseudopotentials for Multicomponent Quantum Hall Systems, Phys. Rev. B 85, 075430 (2012).

[44] C. H. Lee, R. Thomale, and X.-L. Qi, Pseudopotential Formalism for Fractional Chern Insulators, Phys. Rev. B 88, 035101 (2013).

[45] Y.-L. Wu, N. Regnault, and B. A. Bernevig, Bloch Model Wave Functions and Pseudopotentials for All Fractional Chern Insulators, Phys. Rev. Lett. 110, 106802 (2013).

[46] C. H. Lee and X.-L. Qi, Lattice Construction of Pseudopotential Hamiltonians for Fractional Chern Insulators, Phys. Rev. B 90, 085103 (2014).

[47] M. Claassen, C. H. Lee, R. Thomale, X.-L. Qi, and T. P. Devereaux, Position-Momentum Duality and Fractional Quantum Hall Effect in Chern Insulators, Phys. Rev. Lett. 114, 236802 (2015).

[48] F. D. M. Haldane, Geometrical Description of the Fractional Quantum Hall Effect, Phys. Rev. Lett. 107, 116801 (2011).

[49] B. A. Bernevig and F. D. M. Haldane, Model Fractional Quantum Hall States and Jack Polynomials, Phys. Rev. Lett. 100, 246802 (2008).

[50] X.-G. Wen and Z. Wang, Classification of Symmetric Polynomials of Infinite Variables: Construction of Abelian and Non-Abelian Quantum Hall States, Phys. Rev. B 77, 235108 (2008).

[51] F. D. M. Haldane and E. H. Rezayi, Periodic LaughlinJastrow Wave Functions for the Fractional Quantized Hall Effect, Phys. Rev. B 31, 2529 (1985).

[52] F. D. M. Haldane, Many-Particle Translational Symmetries of Two-Dimensional Electrons at Rational Landau-Level Filling, Phys. Rev. Lett. 55, 2095 (1985).

[53] S. A. Trugman and S. Kivelson, Exact Results for the Fractional Quantum Hall Effect with General Interactions, Phys. Rev. B 31, 5280 (1985).

[54] S. H. Simon, E. H. Rezayi, N. R. Cooper, and I. Berdnikov, Construction of a Paired Wave Function for Spinless Electrons at Filling Fraction $\nu=2 / 5$, Phys. Rev. B 75, 075317 (2007).

[55] J. Flavin, R. Thomale, and A. Seidel, Gaffnian Holonomy through the Coherent State Method, Phys. Rev. B 86, 125316 (2012).
[56] A. Seidel and K. Yang, Halperin $\left(m, m^{\prime}, n\right)$ Bilayer Quantum Hall States on Thin Cylinders, Phys. Rev. Lett. 101, 036804 (2008).

[57] B. A. Bernevig and N. Regnault, Anatomy of Abelian and Non-Abelian Fractional Quantum Hall States, Phys. Rev. Lett. 103, 206801 (2009).

[58] D. R. Luhman, W. Pan, D. C. Tsui, L. N. Pfeiffer, K. W. Baldwin, and K.W. West, Observation of a Fractional Quantum Hall State at $\nu=1 / 4$ in a Wide GaAs Quantum Well, Phys. Rev. Lett. 101, 266804 (2008).

[59] Z. Papić, G. Möler, M. Milovanović, N. Regnault, and M. O. Goerbig, Fractional Quantum Hall State at $\nu=1 / 4$ in a Wide Quantum Well, Phys. Rev. B 79, 245325 (2009).

[60] D. Green, Ph.D. thesis, Yale University, New Haven (2001), arXiv:cond-mat/0202455.

[61] M. Hermanns, N. Regnault, B. A. Bernevig, and E. Ardonne, From Irrational to Nonunitary: Haffnian and HaldaneRezayi Wave Functions, Phys. Rev. B 83, 241302 (2011).

[62] S. C. Davenport, E. Ardonne, N. Regnault, and S. H. Simon, Spin-Singlet Gaffnian Wave Function for Fractional Quantum Hall Systems, Phys. Rev. B 87, 045310 (2013).

[63] E. Ardonne, N. Read, E. Rezayi, and K. Schoutens, NonAbelian Spin-Singlet Quantum Hall States: Wave Functions and Quasihole State Counting, Nucl. Phys. B607, 549 (2001).

[64] E. Ardonne and N. Regnault, Structure of Spinful Quantum Hall States: A Squeezing Perspective, Phys. Rev. B 84, 205134 (2011).

[65] R. Thomale, B. Estienne, N. Regnault, and B. A. Bernevig, Decomposition of Fractional Quantum Hall Model States: Product Rule Symmetries and Approximations, Phys. Rev. B 84, 045127 (2011).

[66] B. I. Halperin, Theory of the Quantized Hall Conductance, Helv. Phys. Acta 56, 75 (1983).

[67] S. H. Simon, E. H. Rezayi, and N. Regnault, Quantum Hall Wave Functions Based on $S_{3}$ Conformal Field Theories, Phys. Rev. B 81, 121301 (2010).

[68] X.-G. Wen and A. Zee, Shift and Spin Vector: New Topological Quantum Numbers for the Hall Fluids, Phys. Rev. Lett. 69, 953 (1992).

[69] C. R. Dean, A. F. Young, P. Cadden-Zimansky, L. Wang, H. Ren, K. Watanabe, T. Taniguchi, P. Kim, J. Hone, and K. L. Shepard, Multicomponent Fractional Quantum Hall Effect in Graphene, Nat. Phys. 7, 693 (2011).

[70] Z. Papić, R. Thomale, and D. A. Abanin, Tunable Electron Interactions and Fractional Quantum Hall States in Graphene, Phys. Rev. Lett. 107, 176602 (2011).

[71] C. H. Lee and A. Lucas, Simple Model for Multiple-Choice Collective Decision Making, Phys. Rev. E 90, 052804 (2014). 